\title{
An Ocean Drum: quasi-geostrophic energetics from a Riemann geometry perspective
}

\author{
José Luis Jaramillo \\ Institut de Mathématiques de Bourgogne (IMB), \\ UMR 5584, CNRS, Université de Bourgogne Franche-Comté, F-21000 Dijon, France \\ Laboratoire de Physique des Océans (LPO), \\ UMR 6523, CNRS, Université de Bretagne Occidentale, 29200 Brest, France \\ E-mail: Jose-Luis. Jaramillo@u-bourgogne.fr
}

\begin{abstract}
We revisit the discussion of the energetics of quasi-geostrophic flows from a geometric perspective based on the introduction of an effective metric, built in terms of the flow stratification and the Coriolis parameter. In particular, an appropriate notion of normal modes is defined through a spectral geometry problem in the ocean basin (a compact manifold with boundary) for the associated Laplace-Beltrami scalar operator. This spectral problem can be used to systematically encode non-local aspects of stratification and topography. As examples of applications we revisit the isotropy assumption in geostrophic turbulence, identify (a patch of) the hyperbolic space $\mathbb{H}^{3}$ as the leading-order term in the effective geometry for the deep mesoscale ocean and, finally, discuss some diagnostic tools based on a simple statistical mechanics toy-model to be used in numerical simulations and/or observations of quasi-geostrophic flows.
\end{abstract}

Keywords: quasi-geostrophic equations, spectral geometry, statistical mechanics

\section{Introduction}

Quasi-geostrophic (QG) dynamics describes some of the slow motions of the ocean and atmosphere at mid-high latitudes. Our main goal in this article is to recast QG dynamics in a form appropriate for the application of general Riemann geometrical analysis tools, that can bring insight into generic qualitative aspects of the QG model.

Our main ultimate motivation is the assessment of generic features of stratification and topography in ocean dynamics, in particular in QG dynamics in the mesoscale ocean $(\sim 20-200 \mathrm{~km})[1,2$, 3, 4, 5]. QG dynamics applies generically to rotating (stratified) fluids close to geostrophic equilibrium (characterized by the balance between Coriolis accelerations and horizontal pressure gradients), where horizontal scales are much larger than vertical ones. QG dynamics can then be seen as a quasi-static transition along instantaneous geostrophic equilibria, that filters rapid motions such as internal waves while keeping sufficient dynamical richness to study the slow motions. The latter dominate the energy content at low (subinertial) frequencies [6]. The QG model presents 
remarkable structural properties, namely the conservation of physical quantities such as the energy and enstrophy, that permit to account for non-trivial observed physical phenomena and work as a test-bed for more complex treatments of ocean dynamics. Of particular relevance is the discussion of geostrophic turbulence [7, 8, 9] and its role in the formation of large coherent structures (geostrophic eddies, jets, filaments) through the associated turbulent cascades, similar to the 2-dimensional turbulence mechanism [10, 11, 12]. This explains the success of the QG model, even if it offers a very simplified treatment of ocean dynamics. In spite of the extensive study of QG dynamics, we argue here that there is still room for further refinement by incorporating generic qualitative aspects encoded in the geometric structure of the operators controlling the dynamics.

In the present article we adopt such a geometric approach and start exploring the insights it provides into some qualitative features of ocean mesoscale dynamics. In a first stage, we introduce the basic elements to revisit QG dynamics from a Riemann geometry perspective. In particular, we construct an effective (Riemann) metric from the stratification and Coriolis ingredients of a given (bulk) water mass and recast QG equations in terms of the associated (exact) 3-dimensional scalar Laplacian. We focus then on QG energetics and formulate a Laplacian eigenvalue problem in the ocean basin, where topography enters through the boundary conditions. This spectral geometry problem, that incorporates explicitly stratification and topography elements of the basin (and that we refer to as an ocean drum) opens an avenue to the qualitative analysis of the QG model: spectral geometry tools permit to extract information of the system without the need of explicitly solving the corresponding analytic equations. The presentation of these formal geometric elements constitutes the main goal of this article.

In a second stage, at a more exploratory level, we consider some lines of application in the ocean context (the formal elements above essentially apply also in an atmosphere setting). First, we discuss how the geometric perspective brings further insight into the isotropy assumption in the analysis of geostrophic turbulence, in particular addressing the optimal stratification conditions for its occurrence. Second, we point out the potential role of hyperbolic geometry elements in the study of QG structures in the deep ocean, opening an ocean physics scenario for the transfer and application of geometric results in Riemann spaces of (constant) negative curvature. Third, we present a set of diagnosis tools for the analysis of observational data/numerical simulations of QG flows. Concretely, these qualitative estimators are constructed out of an (ad hoc) statistical mechanics toy model motivated by the ocean drum problem. The specific goal of such a model is not to capture the actual QG physics, but rather to provide a simple approach to map QG field configurations, namely taking into account stratification and topography, through the systematics of the related thermodynamical quantities. The previous three examples constitute a brainstorm exercise on applications of the adopted geometric approach. Regarding a more systematic framework, the Robert-Sommeria-Miller (RSM) [13, 14, 15, 16] equilibrium statistical mechanics theory for 2-dimensional flows provides a powerful formalism for the application of the (spectral) Riemann geometry elements presented here, in particular in the construction of invariant measures for continuously 
stratified QG flows along the lines in [17]. This statistical mechanics objective is an ultimate target of our approach, requiring a specific discussion to be addressed elsewhere.

The plan of the article is the following. Section 2 briefly reviews the fundamentals of QG dynamics and energetics. Section 3 presents the elements of Riemann geometry needed in the rest of the article. These first two sections introduce the basic building blocks, in particular setting the notation. Section 4 contains the main contribution of the article, namely the identification of an effective metric permitting a compact geometric rewriting of the QG elements, in particular through the formulation of a geometric eigenvalue problem (ocean drum) for a given ocean basin. In section 5 , and in contrast with the "mathematical physics" flavour of section 4, we adopt a more heuristic approach and apply (in a "theoretical physics" spirit) the previous geometric elements to the specific discussion in the ocean. Conclusions are presented in section 6. The main text is complemented with five technical appendices. Having in mind a broad reader profile, we make an effort to present an essentially self-contained discussion, perhaps at the price of revisiting elements occasionally too basic for the expert in the field.

\section{Quasi-geostrophic dynamics and energetics}

Let us consider an ocean basin manifold $M$ with given Cartesian coordinates $(x, y, z)$ and an incompressible flow $\mathbf{v}$, with components $\mathbf{v}=u \mathbf{e}_{x}+v \mathbf{e}_{y}+w \mathbf{e}_{z}$ in the Cartesian orthonormal frame. The QG equations for a rotating and stratified fluid at mid and high latitudes are derived from the Navier-Stokes equation in Boussinesq approximation, in an (asymptotic) expansion around the hydrostatic and geostrophic equilibria. As stated above, geostrophic equilibrium is achieved by the balance between rotation (Coriolis) accelerations and horizontal pressure gradients. The geostrophic flow is characterized by a velocity $\mathbf{v}_{g}$, expressed (in the so-called f-plane approximation) as (see e.g. [2])

$$
\mathbf{v}_{g}=\mathbf{e}_{z} \times \nabla \psi
$$

where the streamfunction $\psi$ is given by $\psi=\frac{p}{f_{o} \rho_{o}}$, with $p$ the pressure (anomaly), $\rho_{o}$ a mean reference value for the density and $f_{o}$ the Coriolis parameter at latitude $\varphi_{o}$ (more generally, $f=2 \Omega \sin \varphi$, with $\Omega$ the Earth's rotation frequency and $\varphi$ a given latitude). Therefore the geostrophic $\mathbf{v}_{g}$ defines, at each depth $z$, a 2-dimensional divergence-free flow. QG dynamics is obtained as a small deviation of the geostrophic flow in a regime of fast environmental rotation and strong density stratification. These features are respectively characterized by the smallness of the Rossby (Ro) and Froude (Fr) numbers. The former is defined in terms of a characteristic horizontal velocity $U$, the rotation frequency $f_{o}$ and a horizontal length scale $L$ as $R_{0}=\frac{U}{f_{o} L}$, whereas the latter is given by $\operatorname{Fr}=\frac{U}{N H}$, where $H$ is a vertical length scale and $N$ is the buoyancy or BruntVäisälä frequency accounting for the density stratification $\left(N^{2}=-\frac{g}{\tilde{\rho}} \frac{d \tilde{\rho}}{d z}\right.$, with $g$ the gravitational acceleration and $\tilde{\rho}(z)$ an average density at depth $z$, providing the reference stratification around which the density $\rho$ is linearized: $\rho=\tilde{\rho}+\delta \rho$ with $\delta \rho / \tilde{\rho} \ll 1)$. Under the assumption Ro $\sim \mathrm{Fr}=\epsilon \ll 1$ and expressing the velocity as $\mathbf{v}=\mathbf{v}_{g}+\mathbf{v}_{a g}$, where 
$\mathbf{v}_{a g} \sim o(\epsilon)$ denotes the corrections to the geostrophic velocity, QG equations are obtained from an expansion in $\epsilon$ of an appropriate rescaling of the rotating Boussinesq equations (for formal details of the derivation and further physical insight, including the whole set of assumptions in the QG model, see e.g. [1, 2, 3, 4, 18, 5]). The resulting QG dynamics filters the fast components of the flow, describing the slow motions captured by the conservation of a scalar quantity $q$ referred to as the $Q G$ potential vorticity

$$
q=\Delta_{\|} \psi+\frac{1}{\tilde{\rho}} \partial_{z}\left(\tilde{\rho} \frac{f_{o}^{2}}{N^{2}} \partial_{z} \psi\right)+f
$$

with $\Delta_{\|}=\frac{\partial^{2}}{\partial x^{2}}+\frac{\partial^{2}}{\partial y^{2}}$. In the absence of forcing and dissipation (that can be neglected without loss of generality in the present discussion) QG dynamics is specified by

$$
\partial_{t} q+v_{g}^{i} \nabla_{i} q=0
$$

with $v_{g}^{i}$ the components of the geostrophic velocity in (11). It is customary to write it as

$$
\partial_{t} q+J(\psi, q)=0
$$

where, $J(\psi, q)=\partial_{x} \psi \partial_{y} q-\partial_{y} \psi \partial_{x} q$. The state of the flow is therefore encoded in the streamfunction $\psi$, whose dynamics is dictated by a geostrophic 2-dimensional advection balancing the (vertical component of the) relative vorticity $\left(\zeta=\partial_{x} v-\partial_{y} u=\Delta_{\|} \psi\right.$ ), a vertical-stretching contribution and the planetary vorticity term $f$ [respectively the first, second and third terms in the right-hand-side in Eq. (2)]. QG equations are completed with appropriate boundary conditions, in particular a vanishing vertical (ageostrophic) velocity $\ddagger$, leading to the geostrophic advection of $\partial_{z} \psi$ on the boundary $\partial M$ (e.g. [5])

$$
\left.\left[\partial_{t}\left(\partial_{z} \psi\right)+J\left(\psi, \partial_{z} \psi\right)\right]\right|_{\partial M}=0
$$

This condition is actually devised for the ocean bottom and surface. When considering restricted ocean domains, lateral boundary conditions are also needed. A common choice involves a Dirichlet condition only depending on time, $\left.\psi\right|_{\partial M}=C(t)$. Topography effects are a main interest in the present work, this leading to a focus on condition (5) (cf. however the remark on lateral boundary conditions after the eigenvalue problem in 4.2).

Energetics are of particular relevance in our discussion. The energy $E_{Q G}[\psi]$ of a QG flow with streamfunction $\psi$ contains two terms (e.g. [2]): the kinetic energy of the flow, $E_{\text {kin }}[\psi]=\frac{1}{2} \int_{M}\left|\mathbf{v}_{g}\right|^{2} \tilde{\rho} d^{3} x=\frac{1}{2} \int_{M}\left|\nabla_{\|} \psi\right|^{2} \tilde{\rho} d^{3} x$, and a second term accounting for the so-called available potential (AP) energy, of the form $E_{\mathrm{AP}}[\psi]=\frac{1}{2} \int_{M} \frac{f_{o}^{2}}{N^{2}}\left(\partial_{z} \psi\right)^{2} \tilde{\rho} d^{3} x$. They correspond respectively to the relative vorticity and vertical-stretching terms

$$
E_{Q G}[\psi]=E_{\mathrm{kin}}[\psi]+E_{\mathrm{AP}}[\psi]=\frac{1}{2} \int_{M}\left(\left|\nabla_{\|} \psi\right|^{2}+\frac{f_{o}^{2}}{N^{2}}\left(\partial_{z} \psi\right)^{2}\right) \tilde{\rho} d^{3} x .
$$

Notably the planetary vorticity plays no role in the energetics. Indeed, only the $q-f$ part of the QG potential vorticity $q$ enters into the energy, defining a linear elliptic operator $\hat{L}$ acting on $\psi$, introduced by Charney [7] in the analysis of geostrophic turbulence

$$
\hat{L} \psi \equiv q-f=\Delta_{\|} \psi+\frac{1}{\tilde{\rho}} \partial_{z}\left(\tilde{\rho} \frac{f_{o}^{2}}{N^{2}} \partial_{z} \psi\right)
$$

$\ddagger$ The first contribution to the (ageostrophic) vertical velocity is $w=\frac{f_{o}}{N^{2}}\left[\frac{\partial^{2} \psi}{\partial t \partial z}+J\left(\psi, \partial_{z} \psi\right)\right]$ (e.g. [5]). 
In terms of the operator $\hat{L}$ the QG energy can be rewritten (up to boundary terms) as

$$
E_{Q G}^{\mathrm{T}}[\psi]=-\frac{1}{2} \int_{M} \psi\left[\Delta_{\|}+\frac{1}{\tilde{\rho}} \partial_{z}\left(\tilde{\rho} \frac{f_{o}^{2}}{N^{2}} \partial_{z}\right)\right] \psi \tilde{\rho} d^{3} x=-\frac{1}{2} \int_{M} \psi \hat{L} \psi \tilde{\rho} d^{3} x
$$

This form of the energy as the expectation value of the operator $\hat{L}$ at the "state" given by the streamfunction $\psi$ presents two appealing features. On the one hand, from a technical perspective it suggests to explore the energetics associated with $E_{Q G}[\psi]$ in terms of the analysis of the operator $\hat{L}$, in particular through the systematic study of its spectral properties (the analysis of cascades in geostrophic turbulence [7] is akin to such approach). On the other hand, from a heuristic point of view, the formal resemblance with the energy expectation values in quantum mechanics can be exploited to introduce novel approaches into the problem, complementary to more standard fluid mechanics treatments. As an example, the calculation of energy corrections to a dynamical QG flow, from a given reference one, can benefit from tools in operator perturbation theory.

\subsection{Enstrophy}

Let us define the enstrophy $\mathcal{E}[\psi]$ of a QG flow, given by the streamfunction $\psi$, as

$$
\mathcal{E}[\psi]=\int_{M}(\hat{L} \psi)^{2} \tilde{\rho} d^{3} x
$$

A key feature of QG dynamics is the conservation of $\mathcal{E}[\psi]$ along the QG flow. The existence of this additional invariant plays a key role in geostrophic turbulence [7]. More generally, functionals of the potential vorticity $q$ associated with arbitrary functions $s$

$$
\mathcal{C}_{s}[q]=\int_{M} s(q) d^{3} x
$$

are also preserved by the QG flow. These infinite dynamical invariants, referred to as Casimirs, play a fundamental role in the statistical mechanics of QG flows (e.g. [19]).

\section{Riemannian geometry and Laplace-Beltrami operator}

In this section we collect the elements of Riemannian geometry that we need for our discussion, in particular fixing the notation (see additional details in Appendix A).

\subsection{Some elements of Riemannian geometry}

3.1.1. Metric tensor. Let us consider a Riemannian metric $\mathrm{g}$ defined on a compact $d$-dimensional manifold $M$, possibly with boundaries (we shall focus on $d=3$, but we keep a general $d$ in this section). Given a local chart $\left\{x^{i_{1}}, \ldots, x^{i_{d}}\right\}$, the metric is written as $\mathbf{g}=g_{i j} d x^{i} \otimes d x^{j}$, where $g_{i j}$ is a non-degenerate positive-definite symmetric matrix. The metric line element is written as $d s^{2}=g_{i j} d x^{i} d x^{j}$, with $s$ the arc-length parameter. Let us denote the inverse of the $g_{i j}$ matrix by $g^{i j}$, i.e. $g^{i k} g_{k j}=\delta^{i}{ }_{j}$, and its determinant by $g=\operatorname{det}\left(g_{i j}\right)$. The metric volume form is given by $\boldsymbol{\epsilon}_{g}=\sqrt{g} d x^{1} \wedge \ldots \wedge d x^{d}=$ $\frac{\sqrt{g}}{d !} \epsilon_{i_{1} \ldots i_{d}} d x^{i_{1}} \wedge \ldots \wedge d x^{i_{d}}$, with $\epsilon_{i_{1} \ldots i_{d}}$ the completely antisymmetric tensor with $\epsilon_{1 \ldots d}=1$. 
An Ocean Drum: quasi-geostrophic energetics from a Riemann geometry perspective

3.1.2. Connection and curvature. Let us denote by $\nabla$ the Levi-Civita connection associated with $\mathbf{g}$, i.e. the unique connection that is metric compatible (i.e. $\nabla_{k} g_{i j}=$ 0 ) and torsion free (i.e. $\left(\nabla_{i} \nabla_{j}-\nabla_{j} \nabla_{i}\right) \phi=0$, for all scalar fields $\phi$ on $\left.M\right)$. Geodesics satisfy $u^{j} \nabla_{j} u^{i}=0$, with unit-tangent vector $\mathbf{u}=u^{i} \partial_{x^{i}}=\frac{d x^{i}}{d s} \partial_{x^{i}}$. Regarding its curvature, the Riemann tensor components are denoted by $R_{j k l}^{i}$, where $\left(\nabla_{k} \nabla_{l}-\nabla_{l} \nabla_{k}\right) V^{i}=R_{j k l}^{i} V^{j}$, with $\mathbf{V}=V^{i} \partial_{x^{i}}$ any vector field on $M$. The Ricci tensor is the trace of the Riemann tensor, with components $R_{i j}=R_{i k j}^{k}$, and the Ricci scalar is $R=R_{i}^{i}=g^{i j} R_{i j}$.

3.1.3. Geometry of the boundary $\partial M$. When considering topography, it becomes relevant to control the geometry of the boundary of $M$. Let us consider the boundary $\partial M$ with outgoing normal vector $\mathbf{s}=s^{i} \partial_{x^{i}}$, normalized as $s^{i} s_{i}=g_{i j} s^{i} s^{j}=1$. Let us denote by $\mathbf{h}$ the Riemannian metric induced on $\partial M$ from $\mathbf{g}$. We denote also by $\mathbf{h}$ the orthogonal projector onto $\partial M$, given by $\mathbf{h}=h^{i}{ }_{j} \partial_{x^{i}} \otimes d x^{j}=\left(g^{i}{ }_{j}-s^{i} s_{j}\right) \partial_{x^{i}} \otimes d x^{j}=$ $\left(\delta^{i}{ }_{j}-s^{i} s_{j}\right) \partial_{x^{i}} \otimes d x^{j}$. Denoting by $h$ the determinant of $\mathbf{h}$, the volume (area) form on $\partial M$ is $\boldsymbol{\epsilon}_{h}=\sqrt{h} d x^{1} \wedge \ldots \wedge d x^{d-1}$ (this assumes $\partial M$ given by $x^{d}=$ const). The Levi-Civita connection of $\mathbf{h}$ is denoted by $\mathbf{D}$, i.e. $D_{k} h_{i j}=0$ and $\left(D_{i} D_{j}-D_{j} D_{i}\right) \phi=0$. The extrinsic curvature of $(\partial M, \boldsymbol{h})$ into $(M, \boldsymbol{g})$ is given by the tensor $\mathbf{K}=\left(h^{k}{ }_{i} h_{j}{ }_{j} \nabla_{k} s_{l}\right) d x^{i} \otimes d x^{j}$, defined on $\partial M$. The mean curvature $K$ of $\partial M$ is the trace of $\mathbf{K}$, i.e. $K=h^{i j} K_{i j}=\nabla_{i} s^{i}$.

3.1.4. Laplacian operator. A fundamental object in our discussion is the scalar LaplaceBeltrami operator $\Delta_{g}$ associated with the metric $\mathbf{g}$, that we shall refer to simply as the Laplacian. The action of $\Delta_{g}$ on a scalar $\phi$, is given by the divergence of its gradient

$$
\Delta_{g} \phi=\nabla_{i} \nabla^{i} \phi=g^{i j} \nabla_{i} \nabla_{j} \phi .
$$

Given arbitrary coordinates, it holds the important expression in our present context

$$
\Delta_{g} \phi=\frac{1}{\sqrt{g}} \partial_{x^{i}}\left(\sqrt{g} g^{i j} \partial_{x^{j}} \phi\right) \text {. }
$$

3.1.5. Integration on $M$ and $\partial M$. The volume form $\boldsymbol{\epsilon}_{g}$ permits to integrate a scalar $\phi$ on $M$, as $\int_{M} \phi \boldsymbol{\epsilon}_{g}$. As notation, we introduce the associated integration measure as $\mu_{g}=d V_{g}=\sqrt{g} d^{d} x$. In particular the volume $V_{g}$ of $M$, as measured by $\mathbf{g}$, is

$$
V_{g}=\int_{M} 1 \cdot \epsilon_{g}=\int_{M} \sqrt{g} d^{d} x=\int_{M} d V_{g} .
$$

A scalar product $\langle\cdot, \cdot\rangle_{g}$ is defined on $L^{2}\left(M, \mu_{g}\right)$, as

$$
\langle\phi \mid \psi\rangle_{g} \equiv \int_{M} \phi^{*} \psi d V_{g}=\int_{M} \phi^{*} \psi \sqrt{g} d^{d} x,
$$

for generally complex scalar functions $\phi$ and $\psi$. In particular, the Laplacian $\Delta_{g}$ is self-adjoint when restricted to functions satisfying homogeneous Dirichlet or Neumann conditions (more generally, homogeneous Robin conditions). Given a scalar $\phi$ we define the quantity $E_{g}[\phi]$, referred to as the energy of $\phi$ (also Dirichlet energy [20]), as

$$
E_{g}[\phi] \equiv \int_{M} \nabla_{i} \phi^{*} \nabla^{i} \phi d V_{g}=\int_{M} g^{i j} \nabla_{i} \phi^{*} \nabla_{j} \phi d V_{g}=\int_{M}|\nabla \phi|_{g}^{2} d V_{g} .
$$


We denote by $E_{g}^{\mathrm{T}}$ the expected value of the operator $\left(-\Delta_{g}\right)$ at the "state" $\phi$, i.e.

$$
E_{g}^{\mathrm{T}}[\phi] \equiv\left\langle\phi\left|\left(-\Delta_{g}\right)\right| \phi\right\rangle_{g}=\int_{M} \phi^{*}\left(-\Delta_{g}\right) \phi d V_{g} .
$$

As for $M$, we integrate on $\partial M$ with the volume form $\boldsymbol{\epsilon}_{h}$. Specifically, we introduce the integration measure $d A_{h}=\sqrt{h} d x^{d-1}$. Then we define the quantity $E_{\partial M}[\phi]$ as

$$
E_{g}^{\partial M}[\phi] \equiv-\int_{\partial M} \phi^{*} s^{i} \nabla_{i} \phi d A_{h},
$$

that we shall refer to as boundary energy of $\phi$. Integrating by parts it follows the relation

$$
E_{g}^{\mathrm{T}}[\phi]=E_{g}[\phi]+E_{g}^{\partial M}[\phi] .
$$

One could refer to $E_{g}[\phi]$ as the bulk energy of $\phi$ and then to $E_{g}^{\mathrm{T}}[\phi]$ as its total energy.

\subsection{The spectrum of the Laplacian}

Spectral geometry offers a framework for studying geometrical properties of a manifold $M$ out of the spectrum of specific differential operators defined on $M$ and, the other way around, to address generic properties of operators' spectra from the a priori geometric knowledge of $M$. The spectral study of the Laplacian on compact Riemannian manifolds has been systematically addressed, the drum's problem [21, 22] being a prototype.

We consider the eigenvalue problem of the Laplacian on a compact Riemannian manifold with boundaries, subject to homogeneous Neumann boundary conditions

$$
-\Delta_{g} \phi_{n}=\lambda_{n} \phi_{n} \quad,\left.\left.\quad \partial_{\mathbf{s}} \phi_{n}\right|_{\partial M} \equiv s^{i} \nabla_{i} \phi_{n}\right|_{\partial M}=0
$$

More generally, we could consider a mixed eigenvalue problem with (19) in part of $\partial M$ and homogeneous Dirichlet conditions in the rest of $\partial M[20$. We will focus here on the strict Neumann case (cf. 4.2). Eigenvalues $\lambda_{n}$ are non-negative and can be ordered as

$$
0=\lambda_{0}<\lambda_{1} \leq \lambda_{2} \leq \ldots \leq \lambda_{n} \leq \ldots \rightarrow \infty,
$$

with the associated $\left\{\phi_{n}\right\}$ a complete set of orthogonal eigenfunctions on $L^{2}\left(M, \mu_{g}\right)$ [20].

The exact eigenvalue problem is generically out of reach. The power of the geometric approach is that generic qualitative results can be established, even if an explicit solution is absent. We collect some results of interest (see [20], and [23] for a pedagogical review):

i) Bounds on low eigenvalues: lowest non-vanishing eigenvalues encode large scale information. In particular, lower and upper bounds for $\lambda_{1}$ are given by

$$
\lambda_{1} \geq \frac{1}{4} h_{c}^{2} \quad, \quad \lambda_{1} \lesssim 1 / V_{g}^{\frac{2}{d}}
$$

where $h_{c}$ is the so-called Cheeger constant [20], essentially controlling the largest "diameter" of $M$. A useful variational characterization of $\lambda_{1}$ is given by the Rayleigh quotient [with $\phi \in C^{\infty}(M)$, or more generally the Sobolev space $H^{1}(M)$ ]

$$
\lambda_{1}=\inf _{\left(\phi \neq 0, \int_{M} \phi=0\right)} \frac{\int_{M} \nabla^{i} \phi \nabla_{i} \phi d V_{g}}{\langle\phi \mid \phi\rangle_{g}}=\inf _{\left(\phi \neq 0, \int_{M} \phi=0\right)} \frac{E_{g}(\phi)}{\langle\phi \mid \phi\rangle_{g}} .
$$

The minimum is reached for $\phi=\phi_{1}$ and the characterization can be extended to higher eigenvalues $\lambda_{n}$ by imposing orthogonality of $\phi$ and $\phi_{i<n}$. 
An Ocean Drum: quasi-geostrophic energetics from a Riemann geometry perspective 8

ii) Weyl's law: regarding high-eigenvalue asymptotics and denoting with $N(\lambda)$ the number of eigenvalues $\lambda_{n}$ (including multiplicity) such that $\lambda_{n} \leq \lambda$, it holds

$$
N(\lambda) \sim \frac{B_{d}}{(2 \pi)^{d}} V_{g} \lambda^{\frac{d}{2}} \quad(\lambda \rightarrow \infty),
$$

with $B_{d}$ the volume of the unit ball in $\mathbb{R}^{d}$. The asymptotic expression follows

$$
\lambda_{n} \sim \frac{(2 \pi)^{2}}{B_{d}^{\frac{2}{d}}}\left(\frac{n}{V_{g}}\right)^{\frac{2}{d}} \quad(n \rightarrow \infty) .
$$

iii) Heat kernel expansion: spectral functions of an operator, defined from its spectrum and a (generically complex) parameter, offer a powerful way of encoding operator information. For the Laplacian, a prominent example is given by the heat kernel

$$
K(t)=\sum_{n}^{\infty} e^{-t \lambda_{n}}
$$

namely the trace of the heat equation Green's function. Its asymptotics for $t \rightarrow 0$ can be obtained without an explicit knowledge of the spectrum. It holds [24, 25]

$$
K(t)=\frac{1}{(4 \pi t)^{\frac{d}{2}}}\left(V_{g}+\frac{\sqrt{\pi}}{2} A_{h} t^{\frac{1}{2}}+a_{1} t+b_{1} t^{\frac{3}{2}}+a_{2} t^{2}+\ldots\right) \quad(t \rightarrow 0) .
$$

The heat kernel coefficients $a_{i}$ and $b_{i}$ are completely characterised by the geometry of $M$ and $\partial M$, depending on boundary conditions in the spectral problem (19) [see Appendix B for expressions of the first terms [24, 25], in the Neumann case in (19)].

\section{Quasi-geostrophy from a Riemannian perspective}

Charney's operator $\hat{L}$ in (7) is essentially a Laplacian (indeed, an exact "flat" Laplacian for constant stratification). This plays an important role in geostrophic turbulence [7, 4]. In the light of the above-presented geometric Riemannian elements, we show that this is also the case for an arbitrary stratification (with $z$, but also $(x, y)$ dependence). This leads us to revisit some aspects of the QG dynamics from a Riemannian perspective.

\subsection{Quasi-geostrophic metric}

Let us introduce an effective Riemannian metric $\mathbf{g}$ in an ocean basin $M$, with the aim of relating the operator $\hat{L}$ in (7) to the corresponding metric Laplacian $\Delta_{g}$. Considering standard coordinates $(x, y, z)$, we start by adopting the following Ansatz for the metric

$$
d s^{2}=a^{2}\left(d x^{2}+d y^{2}\right)+b^{2} d z^{2} \quad, \text { that is } g_{i j}=\left(\begin{array}{ccc}
a^{2} & 0 & 0 \\
0 & a^{2} & 0 \\
0 & 0 & b^{2}
\end{array}\right),
$$

where $a$ and $b$ depend on $(x, y, z)$. The inverse matrix $g^{i j}$ and the determinant are

$$
g^{i j}=\left(\begin{array}{ccc}
a^{-2} & 0 & 0 \\
0 & a^{-2} & 0 \\
0 & 0 & b^{-2}
\end{array}\right) \quad, \quad g=a^{4} b^{2} \quad, \quad \sqrt{g}=a^{2} b .
$$


Choosing for $a$ and $b$ the expressions

$$
a=\left(\frac{\tilde{\rho}}{\rho_{o}}\right)\left(\frac{f_{o}}{N}\right) \quad, \quad b=\frac{\tilde{\rho}}{\rho_{o}},
$$

the determinant becomes

$$
\sqrt{g}=\left(\frac{\tilde{\rho}^{3}}{\rho_{o}^{3}}\right)\left(\frac{f_{o}^{2}}{N^{2}}\right),
$$

so that, inserting these elements in the expression (11) for $\Delta_{g}$, we can rewrite $\hat{L}$ as

$$
\hat{L} \psi=\left(\frac{\tilde{\rho}^{2}}{\rho_{o}^{2}}\right)\left(\frac{f_{o}^{2}}{N^{2}}\right) \Delta_{g} \psi=\left(\frac{\rho_{o}}{\tilde{\rho}}\right) \Delta_{g} \psi \sqrt{g} .
$$

In the generic case, there is no choice of functions $a$ and $b$ making $\hat{L}$ the Laplacian of some $\mathbf{g}$ (again, for constant $\tilde{\rho}$ and $N$ this is the flat Euclidean Laplacian, with a constant coordinate rescaling). However, the factor is precisely the good one for the energetics. Indeed, inserting (31) in (8), the energy $E_{Q G}^{\mathrm{T}}$ is exactly recast as an expectation value (for the streamfunction $\psi$ ) of the Laplace operator $\left(-\Delta_{g}\right)$ associated with $\mathbf{g}$, with the natural

Riemannian integration measure $d V_{g}=\sqrt{g} d^{3} x$. This proves the following Lemma.

Lemma 1 (quasi-geostrophic metric). Given a $Q G$ flow in a domain $M$ with Cartesian coordinates $(x, y, z)$, streamfunction $\psi$, density stratification $\tilde{\rho}$ with BruntVäisälä frequency $N$ and Coriolis parameter $f_{o}$, let us define the effective QG metric as

$$
d s^{2}=\left(\frac{\tilde{\rho}^{2}}{\rho_{o}^{2}}\right)\left(\frac{f_{o}^{2}}{N^{2}}\left(d x^{2}+d y^{2}\right)+d z^{2}\right) .
$$

Then Charney's operator $\hat{L}$ is written as

$$
\hat{L} \psi=\left(\frac{\tilde{\rho}^{2}}{\rho_{o}^{2}}\right)\left(\frac{f_{o}^{2}}{N^{2}}\right) \Delta_{g} \psi,
$$

and the $Q G$ energies $E_{Q G}$ and $E_{Q G}^{\mathrm{T}}$ for the streamfunction $\psi$ are respectively given by

$$
E_{Q G}[\psi]=\frac{1}{2} \rho_{o} \int_{M} \nabla^{i} \psi \nabla_{i} \psi d V_{g}=\frac{1}{2} \rho_{o} \int_{M}|\nabla \psi|_{g}^{2} d V_{g}
$$

and

$$
E_{Q G}^{\mathrm{T}}[\psi]=\frac{1}{2} \rho_{o} \int_{M} \psi\left(-\Delta_{g}\right) \psi d V_{g}
$$

\section{Remarks:}

a) The geometric recast of $\hat{L}, E_{Q G}$ and $E_{Q G}^{\mathrm{T}}$ in terms of $\mathbf{g}$ does not require $N$ and $\tilde{\rho}$ (and $f_{o}$ ) to depend only on $z$. Expressions (33), (34) and (35) still hold for general functions $N=N(x, y, z)$ and $\tilde{\rho}=\tilde{\rho}(x, y, z)$ beyond the $\mathrm{QG}$ model. Such a freedom could be of interest, e.g. in the study of related perturbation and stability issues.

b) Denoting by $E_{Q G}^{\partial M}$ the boundary term

$$
E_{Q G}^{\partial M}[\psi] \equiv\left(-\frac{1}{2} \rho_{o}\right) \int_{\partial M} \psi \partial_{\mathbf{s}} \psi d A_{h}
$$


An Ocean Drum: quasi-geostrophic energetics from a Riemann geometry perspective 10 energies $E_{Q G}^{\mathrm{T}}[\psi]$ and $E_{Q G}[\psi]$ are related, through integration by parts, as in (18)

$$
E_{Q G}^{\mathrm{T}}[\psi]=E_{Q G}[\psi]+E_{Q G}^{\partial M}[\psi] .
$$

As shown in the relation (66), the QG energy $E_{Q G}[\psi]$ has a kinetic component and an (available) potential energy one. These are bulk contributions to the QG energy. If we consider $E_{Q G}^{\partial M}[\psi]$ as a boundary $Q G$ energy contribution, we can write

$$
E_{Q G}^{\mathrm{T}}[\psi]=E_{\mathrm{kin}}[\psi]+E_{\mathrm{AP}}[\psi]+E_{Q G}^{\partial M}[\psi],
$$

so that $E_{Q G}^{\mathrm{T}}[\psi]$ accounts for the total $Q G$ energy, justifying the chosen notation. To gain physical intuition on the $E_{Q G}^{\partial M}[\psi]$ term, let us consider a flat bottom profile, so that $\partial_{\mathbf{s}}=-\partial_{z}$ at the bottom. On the other hand, pressure and density anomalies are respectively given by $p^{\prime}=\rho_{o} f_{o} \psi$ and $\rho^{\prime}=-\frac{\rho_{o} f_{o}}{g} \partial_{z} \psi$ (e.g. [5]). This leads to

$$
E_{Q G}^{\partial M}[\psi]=-\frac{g}{\rho_{o} f_{o}^{2}} \int_{\partial M} p^{\prime} \rho^{\prime} d A_{h} .
$$

c) As an academic remark, if we formally consider a negative $N^{2}<0$ (convection), the signature of the metric $\mathrm{g}$ changes from Riemannian to Lorentzian. This would change radically the properties of the metric, in particular involving the propagation of perturbations along null-cones of angle $\arctan \left(|N| / f_{o}\right)$ from the vertical. This is however nonphysical in the QG context, that assumes stable stratification $N^{2}>0$.

Focusing on the QG case, $N=N(z)$, the $\mathrm{QG}$ metric presents the following important geometric feature, namely that angles are not changed from the flat Euclidean case.

Lemma 2 (conformal flatness). If $N=N(z)>0$ the $Q G$ metric is conformally flat. In particular, with the change of variables $d z=\frac{f_{o}}{N(z)} d \zeta$, the $Q G$ metric (32) is written as

$$
d s^{2}=\left(\frac{\tilde{\rho}^{2}}{\rho_{o}^{2}}\right)\left(\frac{f_{o}^{2}}{N^{2}}\right)\left(d x^{2}+d y^{2}+d \zeta^{2}\right) .
$$

Proof: It suffices to note that for a non-vanishing $N=N(z)$, the relation $d z=\frac{f_{o}}{N(z)} d \zeta$ in a domain not including the Equator (where $f_{o}=0$ ) defines a good change of variables. Then the expression (40) follows straightforwardly from (32).

\section{Remark:}

The form (40) makes apparent the conformally-flat character of the QG metric in the $N=N(z)$ case. Other coordinates can be preferred in applications, making convenient to have a coordinate-independent criterion characterizing conformal flatness [therefore testing $N=N(z)$ ]. In the present 3-dimensional case, this is provided by the vanishing of the Cotton tensor or, equivalently the Cotton-York tensor, defined in (D.3) and (D.4).

\subsection{An ocean drum}

The expression of $\hat{L}$ and $E_{Q G}^{\mathrm{T}}$ in terms of $\Delta_{g}$ makes natural the study of the eigenvalue problem (19) for the QG metric (32). The associated eigenfunctions can be chosen to be real $\left(\Delta_{g}\right.$ is self-adjoint). Introducing a constant $\alpha_{o}$ with the dimensions of a geostrophic 
An Ocean Drum: quasi-geostrophic energetics from a Riemann geometry perspective 11 streamfunction, i.e. $\left[\alpha_{o}\right]=(\text { Length })^{2}(\text { Time })^{-1}$, we can normalize these eigenfunctions to provide a natural orthogonal basis $\left\{\psi_{n}\right\}$ for the $\mathrm{QG}$ streamfunctions.

Definition (Neumann ocean drum). Given the QG metric (32), we define a set of (real) eigenfunctions $\left\{\psi_{n}\right\}$ of the associated (Neumann) Laplacian eigenvalue problem

$$
-\Delta_{g} \psi_{n}=\lambda_{n} \psi_{n} \quad,\left.\quad \partial_{\mathbf{s}} \psi_{n}\right|_{\partial M}=0
$$

with normalization given by

$$
\left\langle\psi_{m} \mid \psi_{n}\right\rangle_{g}=\int_{M} \psi_{m} \psi_{n} d V_{g}=\left(\alpha_{o}^{2} V_{g}\right) \delta_{n m} .
$$

The $\left\{\psi_{n}\right\}$ Laplacian eigenfunctions and the corresponding eigenvalues $\lambda_{n}$ will be referred to as ocean drum modes of the basin $M$.

The ocean drum modes $\left\{\psi_{n}\right\}$, with dimensions of QG streamfunctions, constitute an orthogonal basis in $L^{2}\left(M, \mu_{g}\right)$. Any streamfunction $\psi$ in $L^{2}\left(M, \mu_{g}\right)$, in particular satisfying (5) but not necessarily Neumann boundary conditions, is uniquely written as

$$
\psi=\sum_{n}^{\infty} C_{n} \psi_{n}, \text { with } C_{n}=\left\langle\psi \mid \psi_{n}\right\rangle_{g}=\frac{1}{\alpha_{o}^{2} V_{g}} \int_{M} \psi \psi_{n} d V_{g},
$$

with $C_{n}$ dimensionless numbers. The norm of $\psi$ can be expressed as

$$
\|\psi\|_{g}^{2}=\langle\psi \mid \psi\rangle_{g}=\left(\alpha_{o}^{2} V_{g}\right) \sum_{n}\left|C_{n}\right|^{2} .
$$

Remark (mixed ocean drum):

The formulated (Neumann) ocean drum focuses on the vertical boundary condition (5), aiming at contacting QG baroclinic modes (cf. Lemma 3 below). A more general ocean drum incorporating lateral boundary conditions can be formulated in terms of a mixed eigenvalue problem [20]: decomposing $\partial M$ in bottom-surface and lateral components as $\partial M=(\partial M)_{\mathrm{BS}} \cup(\partial M)_{\mathrm{L}}$, set $\left.\partial_{\mathrm{s}} \psi_{n}\right|_{(\partial M)_{\mathrm{BS}}}=0$ and $\left.\psi_{n}\right|_{(\partial M)_{\mathrm{L}}}=0$. The mathematical essential ingredient is the homogeneous nature of the conditions, that guarantees the self-adjointness of $\left(-\Delta_{g}\right)$ and completeness of $\left\{\psi_{n}\right\}$. Physically, it is remarkable that they also recover the spatially constant Dirichlet conditions $\left.\psi_{n}\right|_{(\partial M)_{\mathrm{L}}}=C(t)$ for lateral conditions in (3) (indeed homogeneous up to a global constant, at each $t$ ). We are here interested in ocean basins where the area of the bottom boundary dominates over the lateral one. As an approximation, in the following we will focus on the Neumann ocean drum, which renders easier certain technical aspects (in particular the discussion of heat kernel coefficients in Appendix B). A refined ocean drum is however possible.

The following lemma endows ocean drum modes $\left\{\psi_{n}\right\}$ with a physical content, as a stationary basis (in the precise sense to be specified below) onto which the dynamics and energetics of general QG flows can be decomposed.

Lemma 3 (stationary ocean drum modes). The eigenfunctions $\left\{\psi_{n}\right\}$, with $\left\{\lambda_{n}\right\}$ eigen-values, provide a stationary basis for $Q G$ dynamics and energetics in the following sense: 
An Ocean Drum: quasi-geostrophic energetics from a Riemann geometry perspective 12

i) Dynamics: Eigenfunctions $\left\{\psi_{n}\right\}$ satisfy the $Q G$ equations in the $f$-plane, i.e. for $f=f_{o}$ in the $Q G$ dynamical equation (2). In addition, for constant $N$ and $\tilde{\rho}$ and for a flat bottom, the ocean drum modes reduce to standard baroclinic modes.

ii) Energetics: Defining the fundamental energies $E_{n}$ as the $Q G$ energies $E_{Q G}\left[\psi_{n}\right]$ associated with ocean drum modes $\psi_{n}$, we have (setting $M_{o} \equiv \rho_{o} V_{g}$ )

$$
E_{n} \equiv E_{Q G}\left[\psi_{n}\right]=E_{Q G}^{\mathrm{T}}\left[\psi_{n}\right]=\frac{1}{2} M_{o} \alpha_{o}^{2} \lambda_{n} .
$$

Then, for sufficiently regular streamfunctions $\psi=\sum_{n} C_{n} \psi_{n}$ (namely, such that the series converges in $L^{2}\left(M, \mu_{g}\right)$, but also in $H^{1}(M)$ ) not necessarily satisfying $\left.\partial_{\mathbf{s}} \psi\right|_{\partial M}=0$, the bulk $Q G$ energy $E_{Q G}[\psi]$ is decomposed in ocean drum modes $E_{n}$ as

$$
E_{Q G}[\psi]=\sum_{n}\left|C_{n}\right|^{2} E_{n}
$$

so that the total $Q G$ energy is

$$
E_{Q G}^{\mathrm{T}}[\psi]=\sum_{n}\left|C_{n}\right|^{2} E_{n}+\left(-\frac{1}{2} \rho_{o}\right) \int_{\partial M} \psi \partial_{\mathbf{s}} \psi d A_{h} .
$$

Proof: Regarding point $i$ ) we note first that by construction, since we assumed $N \neq N(t)$ and $\tilde{\rho} \neq \tilde{\rho}(t)$, modes $\psi_{n}$ are time independent, i.e. $\partial_{t} \psi_{n}=0$. On the other hand, for $\psi=\psi_{n}$ (in the f-plane $f=f_{o}$ ) we have $q_{n}=\hat{L} \psi_{n}+f=-\lambda_{n}\left(\frac{\tilde{\rho}^{2}}{\rho_{o}^{2}}\right)\left(\frac{f_{o}^{2}}{N^{2}}\right) \psi_{n}+f_{o}$, where we have used relations (33) and (41). Then $\partial_{t} q_{n}=0$ and, given that $\tilde{\rho}$ and $N$ are only functions of $z$, it holds $J\left(\psi_{n}, q_{n}\right)=-\lambda_{n}\left(\frac{\tilde{\rho}^{2}}{\rho_{o}^{2}}\right)\left(\frac{f_{o}^{2}}{N^{2}}\right) J\left(\psi_{n}, \psi_{n}\right)=0$. Therefore (44) is satisfied and $\psi_{n}$ defines a stationary QG flow. On the other hand, baroclinic modes are eigenfunctions of (the vertical-stretching part of) Charney's operator with boundary conditions given by $\left.\partial_{z} \psi\right|_{\partial M}=0$, consistent with the vanishing vertical velocity $(w=0)$ in the general QG boundary condition (5). In the case of constant $N$ and $\tilde{\rho}$, relation (33) implies $-\Delta_{g}=$ const $\cdot \hat{L}$. In addition, for a flat bottom the outgoing normal at the bottom is $\mathbf{s}=-\partial_{z}$, so that the Neumann condition (41) translates into $\left.\partial_{z} \psi\right|_{\partial M}=0$. Then Laplacian Neumann eigenmodes $\psi_{n}$ in (41) recover the baroclinic eigenfunctions.

Regarding $i i), E_{Q G}\left[\psi_{n}\right]=E_{Q G}^{\mathrm{T}}\left[\psi_{n}\right]$ follows from the Neumann boundary conditions for $\psi_{n}$ and relations (18). Expression of $E_{n}$ in terms of $\lambda_{n}$ follows from (35) and the $\psi_{n}$ normalization. To prove (46) for a streamfunction $\psi$ not necessarily satisfying $\partial_{\mathbf{s}} \psi=0$ at the bottom, we note that the assumption of convergence of the series $\psi=\sum_{n} C_{n} \psi_{n}$ in the $H^{1}$ norm \|\|$_{1}$ (namely in $\|\psi\|_{1}^{2}=\|\psi\|_{L^{2}}^{2}+\|\nabla \psi \cdot \nabla \psi\|_{L^{2}}^{2}$ ), implies in particular

$$
\lim _{N \rightarrow \infty} \int_{M}\left(\nabla_{i} \psi-\sum_{n}^{N} C_{n} \nabla_{i} \psi_{n}\right)\left(\nabla^{i} \psi-\sum_{m}^{N} C_{m} \nabla^{i} \psi_{m}\right) d V_{g}=0 .
$$

Then, for a given finite $N$ we can write

$$
\begin{aligned}
& \int_{M}\left(\nabla_{i} \psi-\sum_{n}^{N} C_{n} \nabla_{i} \psi_{n}\right)\left(\nabla^{i} \psi-\sum_{n}^{N} C_{m} \nabla^{i} \psi_{m}\right) d V_{g} \\
= & \int_{M} \nabla_{i} \psi \nabla^{i} \psi d V_{g}-2 \sum_{n}^{N} C_{n} \int_{M} \nabla_{i} \psi \nabla^{i} \psi_{n} d V_{g}+\sum_{n}^{N} \sum_{m}^{N} C_{n} C_{m} \int_{M} \nabla_{i} \psi_{n} \nabla^{i} \psi_{m} d V_{g}
\end{aligned}
$$


An Ocean Drum: quasi-geostrophic energetics from a Riemann geometry perspective 13

$$
\begin{aligned}
& =\int_{M} \nabla_{i} \psi \nabla^{i} \psi d V_{g}+2 \sum_{n}^{N} C_{n} \int_{M} \psi \Delta_{g} \psi_{n} d V_{g}-\sum_{n}^{N} \sum_{m}^{N} C_{n} C_{m} \int_{M} \psi_{n} \Delta_{g} \psi_{m} d V_{g} \\
& =\int_{M} \nabla_{i} \psi \nabla^{i} \psi d V_{g}-\sum_{n}^{N}\left|C_{n}\right|^{2}\left(\alpha_{o}^{2} V_{g}\right) \lambda_{n},
\end{aligned}
$$

where in the second step we have integrated by parts and used the Neumann conditions on $\psi_{n}$, and the third step uses the eigenvalue relation (19) and normalization (42). Taking the limit $N \rightarrow \infty$, using (48) and multiplying by $\frac{1}{2} \rho_{o}$, we recover the expression (46)) for $E_{Q G}[\psi]$. Expression for $E_{Q G}^{\mathrm{T}}[\psi]$ in (47) follows from (46) and relation (18).

\section{Remarks:}

a) The Laplace operator is guaranteed to be selfadjoint for homogeneous Neumann $\left(\left.\partial_{\mathbf{s}} \psi_{n}\right|_{\partial M}=0\right)$, Dirichlet $\left(\left.\psi_{n}\right|_{\partial M}=0\right)$, mixed or, more generally, Robin boundary conditions $\left[\left.\left(a \psi_{n}+b \partial_{\mathbf{s}} \psi_{n}\right)\right|_{\partial M}=0\right]$. The choice of Neumann ones for the ocean drum basis is justified by the recovery of baroclinic modes in point i) of Lemma 3.

b) The bulk contribution to the energy is positive, $E_{Q G}[\psi] \geq 0$. The ocean drum eigenmodes have vanishing boundary energy contribution, $E_{Q G}^{\partial M}\left[\psi_{n}\right]=0$, due to the Neumann boundary conditions. In particular, it follows that ocean drum modes are all non-negative, $E_{n} \geq 0$ (in general, $\lambda_{n} \geq 0$ in the Laplacian eigenvalue problem with Neumann, cf. (20), Dirichlet or mixed boundary conditions). However this does not exclude streamfunctions $\psi$ with negative total energy $E_{Q G}^{\mathrm{T}}[\psi]<0$ (then $\left.\left.\partial_{\mathbf{s}} \psi\right|_{\partial M} \neq 0\right)$. This is accomplished, e.g., with eigenfunctions $\psi$ with negative eigenvalues. The latter can be constructed with Robin conditions such that $a \cdot b<0$, if the boundary contribution in (47) $E_{Q G}^{\partial M}[\psi]=\frac{1}{2} \rho_{o} \int_{\partial_{M}}\left(\frac{a}{b}\right) \psi^{2} d A_{h}<0$ is sufficiently large. Examples of such negative eigenvalues of the Laplacian can be found in [26].

4.2.1. Modified enstrophy. The enstrophy invariant $\mathcal{E}[\psi]$ is defined in (9) in terms of the square of $\hat{L} \psi$. However its rewriting in terms of $\left(-\Delta_{g}\right)$ is not natural from the spectral perspective of the ocean drum. However, we can introduce a modified enstrophy

$$
\tilde{\mathcal{E}}[\psi] \equiv \int_{M}\left(\frac{\tilde{\rho}^{2}}{\rho_{o}^{2}}\right)^{-1}\left(\frac{f_{o}^{2}}{N^{2}}\right)^{-1}(\hat{L} \psi)^{2} \tilde{\rho} d^{3} x,
$$

also an invariant of the $\mathrm{QG}$ dynamics [since $\tilde{\rho}=\tilde{\rho}(z), N=N(z)$ ], that is rewritten as

$$
\tilde{\mathcal{E}}[\psi]=\rho_{o} \int_{M}\left(\Delta_{g} \psi\right)^{2} d V_{g}
$$

using (331) and (30). This is the natural enstrophy invariant $\S$ to be considered in a spectral discussion, since we have (for $\psi=\sum_{n} C_{n} \psi_{n}$ and up to boundary contributions)

$$
\tilde{\mathcal{E}}[\psi]=M_{o} \alpha_{o}^{2} \sum_{n}\left|C_{n}\right|^{2} \lambda_{n}^{2} .
$$

$\S$ It is noteworthy that, for the kinetic part, this is precisely the expression obtained if using the metric $\mathbf{g}$ in the construction of the vector product employed in the expressions of $\mathbf{v}_{g}$ and the relative vorticity, instead of the flat one. 
An Ocean Drum: quasi-geostrophic energetics from a Riemann geometry perspective 14

\section{Perspectives in ocean physics}

Hitherto, the essential elements in the discussion apply in both the oceanic and the atmospheric setting. In this section we focus on the ocean case. In this context, the assumption $\tilde{\rho}=\rho_{o}$ is a good approximation. The QG metric simplifies then to

$$
d s^{2}=\frac{f_{o}^{2}}{N^{2}}\left(d x^{2}+d y^{2}\right)+d z^{2}=\left(\frac{f_{o}^{2}}{N^{2}}\right)\left(d x^{2}+d y^{2}+d \zeta^{2}\right),
$$

the second (explicitly conformally-flat) form assuming $N=N(z)$ (with $d z=\frac{f_{o}}{N} d \zeta$ ). The effective QG metric is a geometric and physical property of an (ocean mesoscale) mass of water, at a given latitude and with a given stratification characterised by the BruntVäisälä frequency $N$. In addition, the ocean drum modes $\left\{\psi_{n}\right\}$ and their eigenvalues $\left\{\lambda_{n}\right\}$ are a property of a given ocean basin. Such geometric recast can provide insight into relevant QG features, but it is not clear that it will be particularly helpful in the explicit resolution of the QG equations. In contrast, it can prove useful in the study of generic and qualitative properties of QG flows, in particular through the application of tools/concepts from spectral geometry. This section discusses some points in this spirit.

\subsection{Isotropy in geostrophic turbulence}

The isotropy assumption plays an important role in Charney's discussion of geostrophic turbulence [7, in particular for deriving the equipartition of energy between kinetic and available potential energy components. Such hypothesis is justified from the structure of the operator $\hat{L}$, together with reasonable physical assumptions. In the present context, the conformally-flat form of the metric in (53) implies that the (QG) ocean is effectively locally quasi-isotropic in scales over which the factor $\frac{f_{o}^{2}}{N^{2}}$ presents small variations, so that it can be considered constant. The metric is then effectively the isotropic flat metric. The Ricci scalar curvature $R$ of $\mathbf{g}$ provides a natural pointwise scale for local variations of the geometry, through the curvature radius defined as $1 / L_{\text {curvature }}^{2} \equiv|R|$. This definition has the virtue of not depending on the coordinates. Choosing $L_{\text {isotropy }} \sim L_{\text {curvature }}$ as the scale below which the quasi-isotropic approximation holds, and using

$$
R=-6\left(\partial_{z} \ln N\right)^{2}+4 \partial_{z}^{2} \ln N,
$$

following from (C.1) [or applying (D.1) to the conformally-flat form of (53)], we have

$$
L_{\text {isotropy }} \sim \frac{1}{\left|6\left(\partial_{z} \ln N\right)^{2}-4 \partial_{z}^{2} \ln N\right|^{\frac{1}{2}}} .
$$

The isotropy hypothesis is well-established in the context of geostrophic turbulence. In spite of this, the present geometric discussion can help in providing a more systematic framework where physical assumptions are simplified and issues like the criticism in [27] can be naturally assessed. In this sense, it is worth noting that the adopted geometric approach indicates the existence of privileged depths for isotropy (and therefore geostrophic turbulence) characterized by the divergence of the scale of isotropy 
An Ocean Drum: quasi-geostrophic energetics from a Riemann geometry perspective 15

$L_{\text {isotropy }} \rightarrow \infty$. From the expression (55) this condition is fulfilled at depths satisfying

$$
6\left(\partial_{z} \ln N\right)^{2}=4 \partial_{z}^{2} \ln N \text {. }
$$

This equation for $z$ defines optimal $Q G$ depths where isotropy is locally maximized.

5.1.1. Aspect ratio. As a by-product, the expression of the aspect ratio between typical vertical and horizontal scales, respectively $\Delta z \sim L_{\mathrm{V}}$ and $\Delta x \sim L_{\mathrm{H}}$, does not depend on depth. This is apparent in the conformally-flat form (53) of the metric, from which

$$
\frac{\Delta \zeta}{\Delta x}=1 \Rightarrow \frac{L_{\mathrm{V}}}{L_{\mathrm{H}}} \equiv \frac{\Delta z}{\Delta x}=\frac{f_{o}}{N} .
$$

Of course, this is just the standard relation following from dimensional analysis estimations. We simply point out that such a relation is actually "exact" in the QG model, without need of such estimations (at least, when separated from the boundaries).

\subsection{Deep ocean and hyperbolic space $\mathbb{H}^{3}$.}

The typical vertical dependence of the Brunt-Väisälä frequency $N$ displays strong gradients in the upper layers of the ocean and then presents a monotonic slow decay at deeper layers, reaching comparatively small values at the bottom. More specifically, in the deep ocean the $z$-dependence of $N$ is well approximated (e.g. [28, 29]) by an exponential $N \sim N_{o} e^{-|z| / H}$ decay (we assume here a negative $z$, with $z=0$ at the surface). We notice from (54) that the Ricci curvature scalar then becomes a negative constant $R \sim-6 / H^{2}$. This has an interesting geometric meaning, namely indicating the hyperbolic nature of the QG metric at the deep ocean. Integrating the relation $d z=\left(f_{o} / N(z)\right) d \zeta$ with the exponential decay results in $N=\frac{f_{o}}{H} \zeta$. Inserting this linear dependence in $\zeta$ in the conformally-flat form of the QG metric (53), we find

$$
d s^{2}=g_{i j}^{\mathbb{H}^{3}} d x^{i} d x^{j}=\frac{H^{2}}{\zeta^{2}}\left(d x^{2}+d y^{2}+d \zeta^{2}\right),
$$

which is the metric of the hyperbolic space $\mathbb{H}^{3}$ (in two dimensions it would correspond to the Poincaré half-plane). In this specific sense $\mathbb{H}^{3}$, or more precisely a compact patch of $\mathbb{H}^{3}$ with boundaries, provides a 0 -th order approximation of the QG metric $g_{i j}$. Indeed

$$
g_{i j}=\Omega^{2} g_{i j}^{\mathbb{H}^{3}}=(1+o(\zeta)) g_{i j}^{\mathbb{H}^{3}},
$$

where $\Omega \equiv \frac{f_{o}}{N} \cdot \frac{\zeta}{H}$ and we have assumed a dependence $N=\frac{f_{o}}{H} \zeta+o\left(\zeta^{2}\right)$ consistent with the leading-order exponential decay of $N$ in $z$. Then, defining $\tilde{\psi} \equiv \Omega^{-\frac{1}{2}} \psi$ and using the conformal transformation properties of the Laplacian in Appendix D, it holds

$$
\left\langle\tilde{\psi}\left|\left(-\Delta_{g}\right)\right| \tilde{\psi}\right\rangle_{g}=\left\langle\psi\left|\left(-\Delta_{\mathbb{H}^{3}}^{\Omega}\right)\right| \psi\right\rangle_{\mathbb{H}^{3}},
$$

where we have defined

$$
\Delta_{\mathbb{H}^{3}}^{\Omega}=\Delta_{\mathbb{H}^{3}}-\left(\frac{1}{2} \Delta_{\mathbb{H}^{3}} \ln \Omega+\frac{1}{4}|\nabla \ln \Omega|_{\mathbb{H}^{3}}^{2}\right) .
$$

This selfadjoint operator (in the hyperbolic scalar product $\langle\cdot \mid \cdot\rangle_{\mathbb{H}^{3}}$ ) can be diagonalized

$$
-\Delta_{\mathbb{H}^{3}}^{\Omega} \psi_{n}^{\Omega}=\lambda_{n}^{\Omega} \psi_{n}^{\Omega},\left.\quad \partial_{\mathbf{s}} \psi_{n}^{\Omega}\right|_{\partial M}=0,
$$


An Ocean Drum: quasi-geostrophic energetics from a Riemann geometry perspective 16 with $g_{i j}^{\mathbb{H}^{3}} s^{i} s^{j}=1$ and $\left\langle\psi_{n}^{\Omega} \mid \psi_{m}^{\Omega}\right\rangle_{\mathbb{H}^{3}}=\delta_{n m}$. Introducing $\tilde{\psi}_{n}^{\Omega} \equiv \Omega^{-\frac{1}{2}} \psi_{n}^{\Omega}$, it holds

$$
\left\langle\tilde{\psi}_{n}^{\Omega}\left|\left(-\Delta_{g}\right)\right| \tilde{\psi}_{m}^{\Omega}\right\rangle_{g}=\lambda_{n}^{\Omega} \delta_{n m} .
$$

Then, for small perturbations $\Omega \sim 1+\epsilon \omega$ of the metric around $\mathbb{H}^{3}$, we have

$$
-\Delta_{\mathbb{H}^{3}}^{\Omega}=-\Delta_{\mathbb{H}^{3}}+\epsilon \frac{\Delta_{\mathbb{H}^{3}} \omega}{2}+o\left(\epsilon^{2}\right),
$$

and, to first order, the coefficients $\lambda_{n}^{\Omega}$ can be written as

$$
\lambda_{n}^{\Omega}=\lambda_{n}^{\mathbb{H}^{3}}+\epsilon \frac{\left\langle\psi_{n}^{\mathbb{H}^{3}}\left|\frac{1}{2} \Delta_{\mathbb{H}^{3}} \omega\right| \psi_{n}^{\mathbb{H}^{3}}\right\rangle_{\mathbb{H}^{3}}}{\left\langle\psi_{n}^{\mathbb{H}^{3}} \mid \psi_{n}^{\mathbb{H}^{3}}\right\rangle_{\mathbb{H}^{3}}},
$$

where $\lambda_{n}^{\mathbb{H}^{3}}$ and $\psi_{n}^{\mathbb{H}^{3}}$ solve the hyperbolic Laplacian eigenvalue problem

$$
-\Delta_{\mathbb{H}^{3}} \psi_{n}^{\mathbb{H}^{3}}=\lambda_{n}^{\mathbb{H}^{3}} \psi_{n}^{\mathbb{H}^{3}} \quad,\left.\quad \partial_{\mathbf{s}} \psi_{n}^{\mathbb{H}^{3}}\right|_{\partial M}=0
$$

This motivates the systematic study of the Laplacian eigenvalue problem (66) in compact submanifolds (with boundaries) of the hyperbolic space $\mathbb{H}^{3}$ (e.g. [30]).

Finally, this hyperbolic leading-order behaviour could be seen as indicating the existence of more (effective) volume available for QG degrees of freedom in the deep ocean than in the upper layers. Such hyperbolic nature of the QG deep ocean suggests geometric problems, such as: i) the $\mathbb{H}^{3}$ isoperimetric problem and optimal forms in $\mathbb{H}^{3}$ providing qualitative descriptions of QG large coherent structures (e.g. vortices), or ii) the role of (hyperbolically) separating geodesics in the study of QG instabilities.

\subsection{Large and small length scales: $Q G$ asymptotics and the heat kernel}

QG dynamics offers an appropriate description of the slow motions of the ocean in the approximate length-scale range $L \sim 20-200 \mathrm{~km}$. At larger or smaller scales the description of the flow must be modified or complemented with other dynamical elements (limits depend on latitude and are not sharp, e.g. $L \sim 50-500 \mathrm{~km}$ also holds). In particular, planetary-geostrophic equations account for large-scale ocean dynamics, whereas at small scales QG dynamics makes a transition into the sub-mesoscale regime requiring general primitive equations or even full Navier-Stokes for its description. In this setting, a good qualitative control of low and large eigenvalues can provide insights into the mechanisms underlying the transition between these distinct dynamical regimes.

5.3.1. Large scales and small eigenvalues. Low eigenvalues provide upper bounds for large QG scales. The energy inverse-cascade of geostrophic turbulence transfers dynamically the energy injected at a given scale into larger ones. But energy cannot be stocked beyond a given largest scale $L_{\max }^{\mathrm{QG}}$ in $\mathrm{QG}$ modes, since the spectrum is bounded below. In this context, taking $L_{\max }^{\mathrm{QG}} \sim 1 / \sqrt{\lambda_{1}}$, bounds in (21) for the lowest eigenvalue $\lambda_{1}$ provide bounds (in particular an upper bound) for such a largest scale, leading to

$$
\left(f_{o} / N\right)^{\frac{2}{3}}\left(L^{2} H\right)^{\frac{1}{3}} \sim V_{g}^{\frac{1}{3}} \lesssim L_{\max }^{\mathrm{QG}} \lesssim 2 / h_{c} \sim\left(f_{o} / N\right) L_{\max }^{\text {basin }},
$$

where $L$ and $H$ are, respectively, typical horizontal and vertical scales of the basin and

$L_{\max }^{\text {basin }}$ is a "largest diameter" of the latter. This particular estimate of the upper bound of 
An Ocean Drum: quasi-geostrophic energetics from a Riemann geometry perspective 17

$L_{\max }^{\mathrm{QG}}$ in terms of $L_{\max }^{\text {basin }}$ is a very crude one $\prod$ (e.g. for mid-latitudes and a deep ocean, we have $f_{o} \sim 10^{-4} s^{-1}$ and $N \sim 10^{-3} s^{-1}$, so that $\left(f_{o} / N\right) \sim 10^{-1}$ and, with $L_{\max }^{\text {basin }} \sim 5 \cdot 10^{3} \mathrm{~km}$, we find $L_{\max }^{\mathrm{QG}} \sim 5 \cdot 10^{2} \mathrm{~km}$ ). However, finer studies (e.g. [31, 32]) of small eigenvalues $\left(\lambda_{1}, \lambda_{2}, \ldots\right)$ are of much interest for the statistical mechanics description of the largest scales of turbulent geophysical flows. The reason is the condensation of energy in the first modes, a phenomenon occurring in 2-dimensional flows as a consequence of the infinity of dynamical Casimir invariants in (10) (e.g. [33, 17, 19]). When considering only the conservation of energy-enstrophy, all energy is condensed in the first mode $\phi_{1}$. If more invariants are included, the energy is not limited to the first mode but is still concentrated in the first ones. Low eigenvalues are then important when building criteria to assess qualitative properties of these flows, in particular random changes in the topology of quasi-steady/equilibrium flow states, mainly controlled by the domain geometry (e.g. [33, 34, 35]). In sum, this 2-dimensional-flow statistical mechanics setting motivates the systematic spectral geometry study of low eigenvalues of the $\Delta_{g}$ Laplacian, with a particular focus on the assessment of the influence of stratification and topography in the largest-scales dynamics [through the study of curvature and boundary-value effects on $\left(\lambda_{1}, \lambda_{2}, \ldots\right)$. The $\mathbb{H}^{3}$ eigenvalue problem in section 5.2 can provide an operational perturbative avenue to approximate expressions of such first eigenvalues. In particular, we aim at applying this approach to low eigenvalues in the construction of invariant microcanonical measures for continuously stratified QG flows along the lines in [17].

In a more applied spirit, lowest eigenmodes can be heuristically used to construct diagnostic tools to assess the proximity of a QG flow (provided numerically or by observational data) to a transition into a new non-QG dynamical regime at large scales. As an example, defining the length scale $L$ of a flow with streamfunction $\psi$ as

$$
L^{-2} \equiv \frac{2}{3} \cdot \frac{\int_{M} \nabla^{i} \psi \nabla_{i} \psi d V_{g}}{\langle\psi \mid \psi\rangle_{g}}=\frac{2}{3} \cdot \frac{\sum_{n} \lambda_{n}\left|C_{n}\right|^{2}}{\sum_{n}\left|C_{n}\right|^{2}},
$$

the Rayleigh quotient (22) indicates that, as long as the following condition is satisfied

$$
L^{2} \lambda_{1} \leq \frac{3}{2}
$$

there is "room" in the spectrum to accommodate the inverse-cascade flow of QG energy (note that $\lambda_{1}$ is a property of the basin, not of the flow). Given a physical basin, the saturation of such condition $\left(L^{2} \lambda_{1} \sim \frac{3}{2}\right)$ signals the transition into a larger scale regime.

5.3.2. Small scales and spectral asymptotics. The small-scale behaviour is controlled asymptotically by Weyl's law (23). Defining a wave number $k \equiv \sqrt{\lambda}$, a spectral density

\| Note that, given an actual physical basin, we can consider it divided into smaller geometric subbasins, and then study how the flow quantities defined in such sub-basins change according to different degrees of "coarse graining" of the flow. Analysis with such "renormalization" flavour will be generically needed in the consistent definition of qualitative flow estimators considered below in section 5.3 .3

T The factor $\frac{2}{d}=\frac{2}{3}$ is justified in Appendix E.1. Note also the alternative definition $L_{\mathrm{T}}^{-2} \equiv$ $\frac{2}{3} \cdot \frac{\left\langle\psi\left|\left(-\Delta_{g}\right)\right| \psi\right\rangle_{g}}{\langle\psi \mid \psi\rangle_{g}}$, containing information on the behaviour at the basin boundary. 
An Ocean Drum: quasi-geostrophic energetics from a Riemann geometry perspective 18 for large $k$ (per mode $\frac{d N(k)}{d k}$ and per mode and volume element $\frac{d N(k)}{d V d k}$ ) is given by

$$
\frac{d N(k)}{d k} \sim 3 \frac{B_{3}}{(2 \pi)^{3}} V_{g} k^{2} \quad, \quad \frac{d N(k)}{d V d k} \sim 3 \frac{B_{3}}{(2 \pi)^{3}} \frac{f_{o}^{2}}{N^{2}} k^{2},
$$

noting $d V_{g}=\frac{f_{o}^{2}}{N^{2}} d V$. This spectral density can then be used in statistical mechanics studies of the energy spectrum at small scales, by using it to relate $d E(k) / d k$ with the energy per mode $d E / d N$. Rotation and stratification enter through the factor $\left(f_{o}^{2} / N^{2}\right)$. In this context, corrections to the Weyl's law asymptotic leading order coming from boundary and/or curvature terms [36, 37], can play an important role in understanding the role of topography and stratification in the energetics. In the following subsection we consider this issue from a thermodynamical perspective, in the context of a statistical mechanics toy-model based on the asymptotics to the heat kernel.

\subsubsection{Topography, stratification and a thermodynamical approach to ocean diagnosis.} When addressing the quantitative aspects of QG dynamics through the resolution of the associated partial differential equations (PDE), even a simplified model as the QG one requires a numerical treatment. This involves two kinds of problems: i) resolution limitations for sufficiently demanding physical settings (e.g. in global ocean models), and ii) efficient extraction of the relevant dynamical information in the resulting complex field configurations. Both problems, respectively of a priori and a posteriori nature, demand an appropriate characterization of the qualitative properties of the flow. The $a$ posteriori issues also apply for observational data of the mesoscale ocean. We consider now a tentative application of the geometric approach complementary to the numerical $\mathrm{PDE}$ resolution, with a specific interest in the diagnosis of stratification and topography effects in QG flows with structures of small typical scale, as compared to the basin size.

Specifically, we propose a family of thermodynamics-like diagnostic estimators, derived from a statistical mechanics toy-model motivated by quasi-geostrophy (details are in Appendix E). Such a model is not equivalent to the full QG model (see Appendix E.2.4, but aims at covering partial aspects of quasi-geostrophy, with a special emphasis in the incorporation of topographic and stratification effects. This is a methodological choice, namely a simplified analysis where some key QG elements are switched-off (in particular, the crucial enstrophy constraint) in order to isolate the addressed effects. The main goal of such thermodynamical treatment is to define systematically, from the thermodynamical potentials and derived quantities, a set of functionals of the streamfunction $\psi$ that can be used as diagnostics to characterize a given mass of water.

Diagnostics calculation. Given a QG $\psi$, the diagnostic estimators are constructed by first integrating the functions $1,|\psi|^{2}$ and $|\nabla \psi|_{g}^{2}$ in the basin $M$. Specifically, we calculate

$$
\int_{M}|\nabla \psi|_{g}^{2} d V_{g}, V_{g}=\int_{M} d V_{g}, \int_{M}|\psi|^{2} d V_{g}
$$


An Ocean Drum: quasi-geostrophic energetics from a Riemann geometry perspective 19 respectively related to $\left(E, V_{g}, \mathrm{~N}\right)$, namely the energy, the (effective) basin volume and the number of (quasi-)particles [see (E.1)], characterizing the thermodynamical macrostate associated with the statistical mechanics toy-model. Although the diagnostic systematics is based on a thermodynamical formalism, in its practical application we do not need to resort to that underlying justification. We demonstrate here the approach in a particular example, and refer to Appendix E for further insight and details.

In a second step, an effective length-scale $L$ for the field $\psi$ is introduced by inverting

$$
\frac{2}{3} \cdot \frac{\int_{M}|\nabla \psi|^{2} d V_{g}}{\int_{M}|\psi|^{2} d V_{g}}=\frac{1}{L^{2}}\left(1-\frac{\sqrt{\pi}}{6} \frac{A_{h}}{V_{g}(L)} L-\frac{2}{3} \frac{a_{1}}{V_{g}(L)} L^{2}-\frac{b_{1}}{V_{g}(L)} L^{3}+\ldots\right),
$$

with

$$
V_{g}(L) \equiv\left(V_{g}+\frac{\sqrt{\pi}}{2} A_{h} L+a_{1} L^{2}+b_{1} L^{3}+a_{2} L^{4}+\ldots\right) .
$$

This relation applies for flows with QG structures with a "thermalized" lengthscale $L$ much smaller than the basin scale, $L \ll\left(V_{g}\right)^{\frac{1}{3}}$. It generalizes the expression for $L$ in (68) by taking into account stratification and topography terms through the heat kernel coefficients $a_{i}$ and $b_{i}$. The squared-length $L^{2}$ plays the role of an effective (inverse) temperature $T$, so that expression (72) is a $E=E\left(T, V_{g}, \mathrm{~N}\right)$ relation [see justification in (E.17) and (E.18) $)$. The knowledge of $\left(L, V_{g}, \mathrm{~N}\right)$ permits to write a (Helmholtz) free energy $F=F\left(L, V_{g}, \mathrm{~N}\right)$ from which thermodynamical quantities can be systematically derived. In this example we give the expression for an effective pressure $P_{g}$ [cf. (E.14)]

$$
P_{g}=\left(1+\frac{\sqrt{\pi}}{2} \frac{A_{h}}{V_{g}} L+\frac{a_{1}}{V_{g}} L^{2}+\frac{b_{1}}{V_{g}} L^{3}+\frac{a_{2}}{V_{g}} L^{4}+\ldots\right)^{-1} \cdot \frac{\rho_{o}}{2 L^{2}} \cdot \frac{\int_{M}|\psi|^{2} d V_{g}}{V_{g}} .
$$

The interest of such a quantity $P_{g}$ is to provide a qualitative criterion for the (mechanical) equilibrium between two masses of water $A$ and $B$ : if $\left(P_{g}\right)_{A} \neq\left(P_{g}\right)_{B}$, one could expect a reconfiguration of the $\psi$ configuration tending to balance $\left(P_{g}\right)_{A}$ and $\left(P_{g}\right)_{B}$. Similarly, other quantities can be constructed to monitor other types of equilibria and, perhaps more interestingly, conditions for internal stability [e.g. $C_{V}>0$ in (E.21) ]. The violation of such stability conditions could signal the need to incorporate new elements in the dynamical description, in particular offering diagnostic tools for the transition from balanced to imbalanced motions (see e.g. [38, 39], also Appendix E.2.2).

5.3.4. Correlation functions. Information of $\psi$ in the thermodynamical diagnostics above is only gained through (71). A finer set of observables, still for configurations with a "thermal" $L$, can be introduced through the expectation value for a field $\phi$

$$
\langle\phi\rangle_{g}^{L} \equiv \frac{1}{\alpha_{o}^{2} V_{g}} \sum_{i}\left\langle\psi_{i}|\phi| \psi_{i}\right\rangle e^{-L^{2} \lambda_{i}} .
$$

In particular, if we consider expectation values of powers $\psi^{n}$ of the streamfunction $\psi$

$$
\left\langle\psi^{n}\right\rangle_{g}^{L}=\frac{1}{\alpha_{o}^{2} V_{g}} \sum_{i}\left\langle\psi_{i}\left|\psi^{n}\right| \psi_{i}\right\rangle e^{-L^{2} \lambda_{i}},
$$

we can use $\left\langle\psi^{n}\right\rangle_{g}^{L}$ to probe scales of the order $\sim(L / n)$. In particular, still in a $L \ll\left(V_{g}\right)^{\frac{1}{3}}$ regime, we can use the heat kernel expansion to evaluate (75) asymptotically, and 
An Ocean Drum: quasi-geostrophic energetics from a Riemann geometry perspective 20

construct estimators $\delta\left(\psi^{n}\right)$ aiming at probing the length scale at which stratification and topography effects become relevant. For this, we compare $\left\langle\psi^{n}\right\rangle_{g}^{L}$ with $\left\langle\psi^{n}\right\rangle_{\text {flat }}^{L}$, the latter evaluated with a flat metric with $\bar{N}=\int_{M} N d V / \int_{M} d V$ (therefore $V_{\text {flat }}=\frac{f_{o}^{2}}{N^{2}} V$, with $V$ the physical volume) and a flat bottom. Using the coefficients in (B.2)

$$
\delta_{L}\left(\psi^{n}\right) \equiv \frac{\left\langle\psi^{n}\right\rangle_{g}^{L}-\left\langle\psi^{n}\right\rangle_{\text {flat }}^{L}}{\left\langle\psi^{n}\right\rangle_{\text {flat }}^{L}} \sim \frac{b_{1}\left[\psi^{n}\right]}{a_{0}\left[\psi^{n}\right]} L+\frac{a_{1}\left[\psi^{n}\right]}{a_{0}\left[\psi^{n}\right]} L^{2}+\frac{b_{1}\left[\psi^{n}\right]}{a_{0}\left[\psi^{n}\right]} L^{3}+\frac{a_{2}\left[\psi^{n}\right]}{a_{0}\left[\psi^{n}\right]} L^{4}+\ldots,
$$

using the approximation $a_{0} \sim\left(a_{0}\right)_{\text {flat }}$. Such $\delta_{L}\left(\psi^{n}\right)$ can be used to probe stratification and topography contributions, since the latter are encoded in $a_{i}\left[\psi^{n}\right]$ and $b_{i}\left[\psi^{n}\right]$.

\section{Conclusions and perspectives}

We have introduced an effective QG metric $\mathbf{g}$, generically with non-vanishing curvature, that constitutes a physical property of a QG mass of water associated with stratification. This permits to rewrite Charney's QG operator $\hat{L}$ in terms of the associated scalar Laplace-Beltrami operator $\Delta_{g}$ and, in particular, the (total) QG energy is given exactly by the expectation value of $\left(-\Delta_{g}\right)$ in the QG state defined by the streamfunction $\psi$. This has prompted the study of the Laplacian, in particular its spectral properties, through the formulation of an ocean drum problem in the ocean basin, namely the Neumann eigenvalue problem of $\left(-\Delta_{g}\right)$. The latter introduces a class of normal modes $\psi_{n}$ whose vertical structure recovers standard QG barotropic/baroclinic modes for constant BruntVäisälä frequencies and provides a f-plane stationary basis for a spectral decomposition of QG streamfunctions $\psi$. The corresponding eigenvalues $\lambda_{n}$ determine the length scale and energy of each mode. Although the exact determination of $\lambda_{n}$ is generically out of reach, spectral geometry tools can be used to estimate properties of the spectrum in terms of curvature and boundary terms, accounting for stratification and topography. This is the main contribution of the article, namely recasting QG elements in a geometric form that can foster new avenues to QG dynamics complementary to existing ones.

In the specific application to the ocean context, we have used the conformallyflat nature of $\mathbf{g}$ to revisit the isotropy assumption in geostrophic turbulence, proposing the existence of preferred depths with enhanced quasi-isotropy. This can be relevant in the understanding of the vertical structure of mesoscale turbulence. On the other hand, we have shown that the QG geometry of the deep ocean is well approximated by the hyperbolic metric in $\mathbb{H}^{3}$. We should generically expect average negative values of the Ricci scalar curvature, sign changes signaling non-trivial configurations of the QG field. This leads to a perturbative approach for studying deep ocean flows, with the hyperbolic space as the natural non-perturbed state. In this setting, we have emphasized the interest of the hyperbolic Laplacian eigenvalue problem in compact submanifolds of $\mathbb{H}^{3}$ with boundary. Finally, we have discussed a class of global diagnostic quantities for numerical/observational data, with a focus on stratification and topography effects. This is a tentative exploration based on an ad hoc but systematic thermodynamical treatment, whose ultimate interest must be assessed from its usefulness as a tool. 
An Ocean Drum: quasi-geostrophic energetics from a Riemann geometry perspective 21

Regarding next research steps, we are particularly interested in the application of the spectral ocean drum problem to the statistical mechanics study of geophysical flows, in particular using RSM theory. A specific goal will be the explicit construction of an invariant microcanonical measure for the continuously stratified QG model, by adapting the work in [17]. Of special interest will be the study of stratification and topography through their incorporation into the spectral properties of the curved effective-metric Laplacian. A second line of research will be the extension of the presented geometric approach to QG dynamics in order to incorporate fast/slow mode coupling in the setting of the wave-mean flow theory for geophysical fluids. In particular, we will focus on the coupling between near-inertial internal waves and QG flows in the Young-Ben Jelloul model [40] and its refinement incorporating the back-reaction onto the QG field [41].

\section{Acknowledgments.}

It is a pleasure to thank X. Carton, A. Colin de Verdière and R. Scott for their scientific advice, patient guide and support. I thank all members of LPO for the warm atmosphere and particularly the scientific discussions with B. Blanke, T. Capuano, D. Ciani, D. Ferjani, A. Hochet, T. Huck, Q. Jamet, M. Kersalé, B. Lecann, P. L'Hegaret, C. Ménesguen, A. Ponte, G. Roullet, R. Schopp, A.M. Tréguier and C. Vic. I also thank F. Bouchet, T. David, E. Gourgoulhon, B. Legras, T. Levasseur, P.A. Luque, J.P. Nicolas, R.T. Pierrehumbert, J. Vanneste, A. Venaille and B. Young. Finally, I would like to express my deep debt of gratitude towards Sergio Dain. This work is dedicated to his memory.

\section{Appendix A. Coordinate expressions of covariant derivatives and curvature}

We complement section 3 with some more explicit expressions for covariant derivatives and curvature. Given a local chart $\left\{x^{i_{1}}, \ldots, x^{i_{d}}\right\}$, the associated basis for the tangent space (bundle) is given by $\left\{\partial_{x^{i_{1}}}, \ldots, \partial_{x^{i_{d}}}\right\}$, whereas $\left\{d x^{i_{1}}, \ldots, d x^{i_{d}}\right\}$ provides a basis for the cotangent bundle. A $\left(\begin{array}{c}m \\ n\end{array}\right)$ tensor field $\mathbf{T}$ can then be written as $\mathbf{T}=T^{j_{1} \ldots j_{m}} i_{i_{1} \ldots i_{n}} \partial_{x^{j_{1}}} \otimes$ $\ldots \otimes \partial_{x^{j_{m}}} \otimes d x^{i_{1}} \otimes \ldots \otimes d x^{i_{n}}$ (summation over indices is assumed). The covariant derivative of a scalar field $\phi$ is just given by standard partial derivatives $\nabla_{i} \psi=\partial_{x^{i}} \phi$, whereas the covariant derivative of a $\left(\begin{array}{l}1 \\ 0\end{array}\right)$ tensor (i.e. a vector field) $\mathbf{V}=V^{i} \partial_{x^{i}}$ is a $\left(\begin{array}{l}1 \\ 1\end{array}\right)$ tensor $\nabla \mathbf{V}$

$$
\nabla \mathbf{V}=\nabla_{i} V^{j} \partial_{x^{j}} \otimes d x^{i} \text {, with } \nabla_{i} V^{j}=\partial_{x^{i}} V^{j}+\Gamma_{i k}^{j} V^{k},
$$

with the Christoffel symbols $\Gamma_{i j}^{k}$ of the Levi-Civita connection associated with the metric $\mathbf{g}=g_{i j} d x^{i} \otimes d x^{j}$ (the latter, namely a symmetric non-degenerate $\left(\begin{array}{l}0 \\ 2\end{array}\right)$ tensor) given by

$$
\Gamma_{i j}^{k}=\frac{1}{2} g^{k l}\left(\partial_{x^{i}} g_{l j}+\partial_{x^{j}} g_{l i}-\partial_{x^{l}} g_{i j}\right) .
$$

The coordinate components of the $\left(\begin{array}{l}1 \\ 3\end{array}\right)$ Riemann tensor introduced in section 3.1 .2 are

$$
R_{j k l}^{i}=\partial_{x^{k}} \Gamma_{j l}^{i}-\partial_{x^{l}} \Gamma_{j k}^{i}+\Gamma_{k m}^{i} \Gamma_{j l}^{m}-\Gamma_{l m}^{i} \Gamma_{j k}^{m} .
$$

The Ricci curvature tensor is a symmetric $\left(\begin{array}{l}0 \\ 2\end{array}\right)$ tensor whose components are obtained by contracting indices in the Riemann tensor as $R_{i j}=R_{i k j}^{k}$. The Ricci scalar is obtained 
An Ocean Drum: quasi-geostrophic energetics from a Riemann geometry perspective 22

by first forming a $\left(\begin{array}{l}1 \\ 1\end{array}\right)$ tensor from the Ricci tensor by "raising" indices with the metric, i.e. $R_{j}^{i}=g^{i k} R_{k j}$, and then contracting the remaining indices: $R=R_{i}^{i}=g^{i j} R_{i j}$.

A property of $\nabla$ playing a crucial role in the geometric rewriting of Charney's operator in section 4 , is the fact that the divergence of a vector [trace of $\nabla \mathbf{V}$ in (A.1)] can be expressed in terms of partial derivatives without the explicit appearance of the Christoffel symbols $\Gamma_{i j}^{k}$. Using (A.2) to rewrite $\Gamma_{k i}^{k}=\frac{1}{2} g^{k j} \partial_{x^{i}} g_{k j}=\partial_{x^{i}} \ln \sqrt{g}$, it follows

$$
\nabla_{i} V^{i}=\partial_{x^{i}} V^{i}+\Gamma_{i k}^{i} V^{k}=\frac{1}{\sqrt{g}} \partial_{x^{i}}\left(\sqrt{g} V^{i}\right) \text {. }
$$

The Laplacian of a scalar field $\phi$ is introduced [Eq. (11)] as the divergence of its gradient, $\Delta_{g} \phi=\nabla_{i} \nabla^{i} \phi$. Writing the components of the gradient of $\phi$ as $\nabla^{i} \phi=g^{i j} \nabla_{j} \phi=g^{i j} \partial_{x^{j}} \phi$ and applying (1.4) to $\nabla^{i} \phi$, it follows the expression (12) for $\Delta_{g} \phi$. Likewise for $K=\nabla_{i} s^{i}$.

\section{Appendix B. Heat kernel coefficients for Neumann boundary conditions}

Given the Laplacian eigenvalue problem (19) with Neumann homogeneous boundary conditions and given a scalar $\phi \in C^{\infty}(M)$, it holds [24, 25] (for normalized $\left\langle\tilde{\psi}_{i} \mid \tilde{\psi}_{j}\right\rangle_{g}=\delta_{i j}$ )

$$
\operatorname{Tr}_{L^{2}}\left(\phi e^{-t\left(-\Delta_{g}\right)}\right)=\sum_{i}\left\langle\tilde{\psi}_{i}|\phi| \tilde{\psi}_{i}\right\rangle_{g} e^{-t \lambda_{i}} \sim \frac{1}{(4 \pi t)^{\frac{d}{2}}}\left(\sum_{n=0} a_{n}[\phi] t^{n}+\sum_{n=0} b_{n}[\phi] t^{\frac{2 n+1}{2}}\right) \quad(t \rightarrow(\mathbb{B}), 1,)
$$

with the first coefficients given by

$$
\begin{aligned}
a_{0}[\phi]= & \int_{M} \phi d V_{g}, \\
b_{0}[\phi]= & \frac{\sqrt{\pi}}{2} \int_{\partial M} \phi d A_{h}, \\
a_{1}[\phi]= & \frac{1}{6}\left(\int_{M} \phi R d V_{g}+\int_{\partial M}\left(2 \phi K-3 \partial_{\mathbf{s}} \phi\right) d A_{h}\right), \\
b_{1}[\phi]= & \frac{\sqrt{\pi}}{2} \frac{1}{96} \int_{\partial M}\left[\phi\left(16 R+8 R_{i j} s^{i} s^{j}+13 K^{2}+2 K_{i j} K^{i j}\right)-6 \partial_{\mathbf{s}} \phi K+24 \partial_{\mathbf{s}} \partial_{\mathbf{s}} \phi\right] d A_{h}, \\
a_{2}[\phi]= & \frac{1}{360}\left[\int_{M} \phi\left(12 \nabla^{i} \nabla_{i} R+5 R^{2}-2 R_{i j} R^{i j}+2 R_{i j k l} R^{i j k l}\right) d V_{g}\right. \\
& +\int_{\partial M} \phi\left(-42 \partial_{\mathbf{s}} R+24 D^{i} D_{i} K+20 R K+4 R_{i j} s^{i} s^{j} K\right. \\
& -12 R_{i k j l} s^{k} s^{l} K^{i j}+4 R_{i j} K^{i j}+\frac{40}{3} K^{3}+8 K_{i j} K^{i j} K+\frac{32}{3} K^{i}{ }_{j} K^{j}{ }_{k} K^{k}{ }_{i} \\
& \left.\left.-\partial_{\mathbf{s}} \phi\left(30 R+12 K^{2}+12 K_{i j} K^{i j}\right)+24 \partial_{\mathbf{s}} \partial_{\mathbf{s}} \phi K-30 \partial_{\mathbf{s}} \nabla^{i} \nabla_{i} \phi\right) d A_{h}\right] .
\end{aligned}
$$

Heat kernel asymptotics are recovered with $\phi=1$. Denoting $a_{i}[1]=a_{i}$ and $b_{i}[1]=b_{i}$

$$
K(t)=\sum_{i} e^{-t \lambda_{i}} \sim \frac{1}{(4 \pi t)^{\frac{d}{2}}}\left(\sum_{n=0} a_{n} t^{n}+\sum_{n=0} b_{n} t^{\frac{2 n+1}{2}}\right) \quad(t \rightarrow 0) .
$$


An Ocean Drum: quasi-geostrophic energetics from a Riemann geometry perspective 23

\section{Appendix C. Curvature elements of the quasi-geostrophic metric}

We collect some curvature elements of the QG metric (53), needed in (B.2). The Ricci scalar, the square of the Ricci tensor and the Kretschmann scalar are, respectively

$$
\begin{aligned}
& R=-\frac{2}{N^{2}}\left(5\left(\frac{\partial N}{\partial z}\right)^{2}-2 N \frac{\partial^{2} N}{\partial z^{2}}\right), \\
& R_{i j} R^{i j}=\frac{2}{N^{4}}\left(17\left(\frac{\partial N}{\partial z}\right)^{4}-14 N\left(\frac{\partial N}{\partial z}\right)^{2} \frac{\partial^{2} N}{\partial z^{2}}+3 N^{2} \frac{\partial^{2} N^{2}}{\partial z^{2}}\right), \\
& R_{i j k l} R^{i j k l}=\frac{4}{N^{4}}\left(9\left(\frac{\partial N}{\partial z}\right)^{4}-8 N\left(\frac{\partial N}{\partial z}\right)^{2} \frac{\partial^{2} N}{\partial z^{2}}+2 N^{2} \frac{\partial^{2} N^{2}}{\partial z^{2}}\right),
\end{aligned}
$$

where $N=N(z)$ is assumed. We note that they are independent of $f_{o}$, depending only on $N$. It is interesting to remark that a dependence in the latitude enters if we relax the QG stratification to a general $N=N(x, y, z)$. We have then, e.g. for the Ricci scalar

$R=-\frac{2\left(N^{2}\left(\frac{\partial N}{\partial x}\right)^{2}-N^{3} \frac{\partial^{2} N}{\partial x^{2}}+N^{2}\left(\frac{\partial N}{\partial y}\right)^{2}-N^{3} \frac{\partial^{2} N}{\partial y^{2}}+5 f_{o}^{2}\left(\frac{\partial N}{\partial z}\right)^{2}-2 f_{o}^{2} N \frac{\partial^{2} N}{\partial z^{2}}\right)}{f_{o}^{2} N^{2}}$

Regarding the extrinsic metric terms of the bottom boundary in $\partial M$, let us consider a topography profile $\eta=\eta(x, y)$. Then, the (outgoing) normal vector is

$$
\mathbf{s}=\frac{1}{\sqrt{\left(\left(\frac{\partial \eta}{\partial x}\right)^{2}+\left(\frac{\partial \eta}{\partial y}\right)^{2}\right) \frac{N^{2}}{f_{o}^{2}}+1}}\left(\frac{N^{2}}{f_{o}^{2}} \cdot \frac{\partial \eta}{\partial x} \frac{\partial}{\partial x}+\frac{N^{2}}{f_{o}^{2}} \cdot \frac{\partial \eta}{\partial y} \frac{\partial}{\partial y}-\frac{\partial}{\partial z}\right),
$$

from where it follows

$$
\partial_{\mathbf{s}} R=-\frac{4\left(5 f_{o}\left(\frac{\partial N}{\partial z}\right)^{3}-6 f_{o} N \frac{\partial N}{\partial z} \frac{\partial^{2} N}{\partial z^{2}}+f_{o} N^{2} \frac{\partial^{3} N}{\partial z^{3}}\right)}{N^{3} \sqrt{\left(\left(\frac{\partial \eta}{\partial x}\right)^{2}+\left(\frac{\partial \eta}{\partial y}\right)^{2}\right) N^{2}+f_{o}^{2}}} .
$$

If we consider the corresponding extrinsic curvature $K_{i j}$, its trace $K$ has the form

$$
\begin{aligned}
K & =\frac{-1}{f_{o} N\left(\left(\frac{\partial \eta}{\partial x}^{2}+{\frac{\partial \eta^{2}}{\partial y}}^{2}\right) N^{2}+f_{o}^{2}\right)^{\frac{3}{2}}}\left[\left(2 \frac{\partial \eta}{\partial x} \frac{\partial^{2} \eta}{\partial x \partial y} \frac{\partial \eta}{\partial y}-\frac{\partial^{2} \eta}{\partial x^{2}} \frac{\partial \eta^{2}}{\partial y} \frac{\partial \eta^{2}}{\partial x} \frac{\partial^{2} \eta}{\partial y^{2}}\right) N^{5}\right. \\
& \left.-\left(f_{o}^{2} \frac{\partial^{2} \eta}{\partial x^{2}}+f_{o}^{2} \frac{\partial^{2} \eta}{\partial y^{2}}\right) N^{3}-\left(2 f_{o}^{4}+3\left(f_{o}^{2} \frac{\partial \eta^{2}}{\partial x}+f_{o}^{2} \frac{\partial \eta^{2}}{\partial y}\right) N^{2}\right) \frac{\partial N}{\partial z}\right] . \quad \text { (C.7) }
\end{aligned}
$$

Similar (but much longer expressions) hold for $K_{i j} K^{i j}$ and $K^{i}{ }_{j} K^{j}{ }_{k} K^{k}{ }_{i}$.

\section{Appendix D. Conformal transformations}

Let us consider two metrics $\mathbf{g}$ and $\tilde{\mathbf{g}}$ on a $d$-dimensional manifold $M$, conformally related as $\tilde{g}_{i j}=\Omega^{2} g_{i j}$. Then, their Ricci scalar curvatures are related as (see e.g. [42])

$$
\tilde{R}=\Omega^{-2}\left[R-2(d-1) g^{i j} \nabla_{i} \nabla_{j} \ln \Omega-(d-2)(d-1) g^{i j} \nabla_{i} \ln \Omega \nabla_{j} \ln \Omega\right] .
$$


An Ocean Drum: quasi-geostrophic energetics from a Riemann geometry perspective 24

On the other hand, given a scalar $\phi$ on $M$ and defining a conformally transformed scalar with conformal weight $s$ as $\tilde{\phi}=\Omega^{s} \phi$, it holds for the respective Laplacians

$$
\begin{aligned}
\Delta_{\tilde{g}} \tilde{\phi}= & \Omega^{s-2} \Delta_{g} \phi+(2 s+d-2) \Omega^{s-3} g^{i j} \nabla_{i} \Omega \nabla_{j} \phi \\
& +s \Omega^{s-3} \phi \Delta_{g} \Omega+s(d+s-3) \Omega^{s-4} \phi g^{i j} \nabla_{i} \Omega \nabla_{j} \Omega .
\end{aligned}
$$

In dimensions $d \geq 4$, the vanishing of the Weyl tensor (traceless part of the Riemann tensor, e.g. [42]) characterizes a given metric as conformally flat. However in dimension $d=3$ the Weyl tensor identically vanishes. Conformal flatness is then characterized by the vanishing of the Cotton tensor that, in dimension $d$, is given by

$$
C_{i j k}=\nabla_{k} R_{i j}-\nabla_{j} R_{i k}+\frac{1}{2(d-1)}\left(\nabla_{j} R g_{i k}-\nabla_{k} R g_{i j}\right) .
$$

The same information is contained in the (Hodge-)dual Cotton-York tensor

$$
C_{i}^{j}=\nabla_{k}\left(R_{l i}-\frac{1}{4} R g_{l i}\right) \epsilon^{k l j} .
$$

Then $C_{i j k}$ and $C_{i}^{j}$ vanish for the conformally-flat QG metric (40) (with $N=N(z)$ ). Deviations from QG, $N=N(x, y, z)$, can be invariantly characterized by the eigenvalues of the symmetric trace-free $C_{i j}$ or, equivalently, by its principal invariants (characteristic polynomial coefficients): $\iota_{2}=-\frac{1}{2} C_{i j} C^{i j}$ and $\iota_{3}=\frac{1}{3} C^{i}{ }_{j} C^{j}{ }_{k} C^{k}{ }_{i}\left(\right.$ since $\left.\iota_{1}=C_{i}{ }^{i}=0\right)$.

\section{Appendix E. A weakly-interacting-particle "QG model"}

Given a flow subject to QG dynamics, let us introduce the following "particle" toymodel. Let us consider a set of $\mathrm{N}$ identical systems, referred to as elementary $Q G$ excitations or $Q G$ quasi-particles, distributed over (stationary) energy levels $E_{n}$ given by (45), i.e. $E_{i}=\frac{1}{2} M_{o} \alpha_{o}^{2} \lambda_{i}$ (with $M_{o}=\rho_{o} V_{g}$ a constant in the model). For a given QG state characterised by $\psi=\sum_{i} C_{i} \psi_{i}$, let us define the level-occupation numbers $\left(n_{1}, n_{2}, \ldots, n_{i}, \ldots\right)$ and the total number $\mathrm{N}$ of $\mathrm{QG}$ quasi-particles as

$$
\mathrm{N} \equiv \frac{\int_{M}|\psi|^{2} d V_{g}}{\alpha_{o}^{2} V_{g}}=\frac{\langle\psi \mid \psi\rangle_{g}}{\alpha_{o}^{2} V_{g}} \quad, \quad n_{i} \equiv\left|C_{i}\right|^{2},
$$

where $\alpha_{o}$ is chosen such that $\mathrm{N} \gg 1$ (the present discussion does not need to fix $\alpha_{o}$ ). Then, denoting $E=E_{Q G}[\psi]$ and using expression (46) and the norm (44) of $\psi$

$$
E=\sum_{i} n_{i} E_{i}, \quad \mathrm{~N}=\sum_{i} n_{i} .
$$

These relations correspond to the distribution of $\mathrm{N}$ (identical) particles with total energy $E$ among the energy levels $E_{i}$ of a mono-particle (quantum) Hamiltonian. Expressions (E.2) are a reminiscent of the (quantum) statistical mechanics treatment $\square$ of the ideal gas, where the monoparticle Hamiltonian is proportional to the Laplacian $\left(-\Delta_{g}\right) *$.

+ A key feature for writing (E.2) is the absence of normalization of $\psi$, a fundamental difference with quantum mechanics that allows us to interpret the (squared) norm of $\psi$ as the total number of particles. * Indeed, this analogy is motivated by the identification [43] of $E_{Q G}[\psi]$ in (6) as a QG classical (noncanonical) Hamiltonian, together with its rewriting (35) as an exact Laplacian (up to boundary terms). Expressions (E.2) then stand in the spirit of a formal Fock "second quantization" of $\psi$ as a free field. 
An Ocean Drum: quasi-geostrophic energetics from a Riemann geometry perspective 25

Of course there are fundamental differences between a QG flow and the ideal gas, in particular regarding the existence of further constraints among the $E_{i}^{\prime} s$. Whereas for weakly interacting particles (strictly non-interacting in the ideal gas) expressions (E.2) encode the whole information, the infinite number of conserved quantities (10) in QG dynamics introduce further constraints. In particular, the enstrophy conservation imposes $\mathcal{E} \sim \sum_{i} n_{i} E_{i}^{2}$ [cf. expression (52)]. This introduces non-local and non-linear interactions among the modes, crucial in geostrophic turbulence. In the present particle toy-model we make the drastic assumption of neglecting such non-local interactions.

Definition (particle toy-model). Given a basin with ocean-drum modes $\left\{\psi_{i}\right\}$ and a $Q G$ flow with $\psi=\sum_{i} C_{i} \psi_{i}$, we define a (microcanonical ensemble) statistical mechanics model of $\mathrm{N}=\langle\psi \mid \psi\rangle_{g} /\left(\alpha_{o}^{2} V_{g}\right)$ "weakly-interacting" identical particles, with total energy $E=E_{Q G}[\psi]$, distributed in the (mono-particle) ocean-drum levels $E_{i}$ with occupation numbers $n_{i}=\left|C_{i}\right|^{2}$, such that $E=\sum_{i} n_{i} E_{i}, \mathrm{~N}=\sum_{i} n_{i}$ hold without further constraints.

\section{Remarks:}

a) This is an ad hoc statistical mechanics model motivated, but different, from QG dynamics. It will be ultimately justified if it provides new insights into aspects of the QG model.

b) Although interactions arising from constraints are eliminated, the model still contains non-local interactions related to topography and stratification (see below). Methodologically, this offers an avenue to isolate and assess the effects of topography and stratification, before "switching on" the rest of actual physical interactions.

c) The model adopts an equilibrium statistical mechanics treatment, a further assumption to be assessed in different particular regimes of QG dynamics.

\section{Appendix E.1. Partition function and free energy: heat kernel expansion.}

Systems with long-range interactions, such as 2-dimensional fluids, display subtle issues concerning statistical ensemble equivalence (e.g. [44, 45, 19]). In the present toy-model we shall relax such concerns and will adopt a canonical ensemble treatment, assuming equilibrium with a thermal bath with formal "temperature" $T$. Such temperature is not the (molecular agitation) physical one, but rather characterizes an equilibrium parameter of the (weakly-interacting) QG particles. As argued below, it is related to a length scale $L$ in a regime of QG dynamics where coherent structures have approximately the same size $L$, much smaller than the basin one. The canonical partition function $Z\left(T, V_{g}, \mathrm{~N}\right)$ of a system of $\mathrm{N}$ weakly-interacting identical particles can be written as

$$
Z\left(T, V_{g}, \mathrm{~N}\right)=\frac{1}{\mathrm{~N} !} Z_{\Delta_{g}}\left(T, V_{g}\right)^{\mathrm{N}},
$$

with $Z_{\Delta_{g}}\left(T, V_{g}\right)$ the mono-particle partition function

$$
Z_{\Delta_{g}}\left(T, V_{g}\right)=\sum_{i} e^{-\beta E_{i}}=\sum_{i} e^{-\frac{E_{i}}{k_{\mathrm{B}} T}}=\sum_{i} e^{-\frac{M_{o} \alpha_{o}^{2}}{2 k_{\mathrm{B}} T} \lambda_{i}},
$$


An Ocean Drum: quasi-geostrophic energetics from a Riemann geometry perspective 26

where $\beta=1 /\left(k_{\mathrm{B}} T\right)$, with $k_{\mathrm{B}}$ the Boltzmann constant. Introducing the length scale $L$ (the relation with the length scale introduced in (68) will be clarified below)

$$
L^{2} \equiv \frac{M_{o} \alpha_{o}^{2}}{2 k_{\mathrm{B}} T}
$$

we have

$$
Z_{\Delta_{g}}\left(L, V_{g}\right)=\sum_{i} e^{-L^{2} \lambda_{i}}, Z\left(L, V_{g}, \mathrm{~N}\right)=\frac{1}{\mathrm{~N} !}\left(\sum_{i} e^{-L^{2} \lambda_{i}}\right)^{\mathrm{N}} .
$$

Hitherto the expression of $Z\left(T, V_{g}, \mathrm{~N}\right)$ is exact in our toy-model. To take a step further and express it in terms of geometric features of $\mathbf{g}$, we can use the heat kernel expansion for small scales $L \rightarrow 0$ (namely $\frac{L}{V_{g}^{1 / 3}} \sim \frac{L}{L_{\text {maxin }}^{\text {basin }}} \ll 1$ ). Making $t=L^{2}$ in (26) (or (B.3))

$$
Z\left(L, V_{g}, \mathrm{~N}\right)=\frac{1}{\mathrm{~N} !} \frac{1}{\left(4 \pi L^{2}\right)^{\frac{3 \mathrm{~N}}{2}}} V_{g}(L)^{\mathrm{N}},
$$

with

$$
V_{g}(L) \equiv\left(V_{g}+\frac{\sqrt{\pi}}{2} A_{h} L+a_{1} L^{2}+b_{1} L^{3}+a_{2} L^{4}+\ldots\right),
$$

where coefficients $a_{i}$ and $b_{i}$ are given in Appendix B (in (B.2) with $\phi=1$ ). Heat kernel coefficients encode curvature (in $a_{i}$ and $b_{i}$ ) and boundary terms (in $b_{i}$ ), bringing stratification and topography interactions, respectively, into the partition function. The non-divergent part of the asymptotic series is analytic in $L$. It is also convenient to express the expansion of $Z$ in terms of the effective temperature $T$, using (E.5)

$$
Z\left(T, V_{g}, \mathrm{~N}\right)=\frac{1}{\mathrm{~N} !}\left(\frac{k_{\mathrm{B}} T}{2 \pi M_{o} \alpha_{o}^{2}}\right)^{\frac{3 \mathrm{~N}}{2}} V_{g}(T)^{\mathrm{N}}
$$

where now (note that the non-divergent part of the expansion is not analytic in $1 / T$ )

$$
V_{g}(T) \equiv\left(V_{g}+\frac{\sqrt{\pi}}{2} A_{h}\left(\frac{M_{o} \alpha_{o}^{2}}{2 k_{\mathrm{B}} T}\right)^{\frac{1}{2}}+a_{1} \frac{M_{o} \alpha_{o}^{2}}{2 k_{\mathrm{B}} T}+b_{1}\left(\frac{M_{o} \alpha_{o}^{2}}{2 k_{\mathrm{B}} T}\right)^{\frac{3}{2}}+\ldots\right) .
$$

Since $\int_{M}|\psi|^{2} d V_{g}$ is not preserved by the QG flow, the grand-canonical ensemble $\Xi$ (where the number of particles is not preserved) is a natural statistical ensemble. It is given by

$$
\Xi\left(T, V_{g}, \mu\right)=\sum_{\mathrm{N}} \sum_{i} e^{\frac{-1}{k_{\mathrm{B}} T}\left(E_{\mathrm{N}, i}-\mu \mathrm{N}\right)}=\sum_{\mathrm{N}} e^{\frac{\mu \mathrm{N}}{k_{\mathrm{B}} T}} Z\left(T, V_{g}, \mathrm{~N}\right),
$$

with $E_{\mathrm{N}, i}$ the energy of the state with $\mathrm{N}$ particles and $\mu$ the chemical potential. We shall give a more explicit expression below (for small $L$ ), using canonical ensemble elements.

\section{Appendix E.2. Thermodynamics elements.}

The (Helmholtz) free energy $F=E-T S$ is built from the canonical partition function as

$$
F=-k_{\mathrm{B}} T \ln Z\left(T, V_{g}, \mathrm{~N}\right)=-\mathrm{N} k_{\mathrm{B}} T\left(\frac{3}{2} \ln T+\ln \left(\frac{V_{g}(T)}{\mathrm{N}}\right)+\text { const }\right),
$$


An Ocean Drum: quasi-geostrophic energetics from a Riemann geometry perspective 27 from which thermodynamical quantities can be systematically derived. First derivatives

$$
S=-\left(\frac{\partial F}{\partial T}\right)_{\left(V_{g}, \mathrm{~N}\right)}, P_{g}=-\left(\frac{\partial F}{\partial V_{g}}\right)_{(T, \mathrm{~N})}, \mu=\left(\frac{\partial F}{\partial N}\right)_{(T, \mathrm{~N})},
$$

provide the entropy $S$, an effective pressure $P_{g}$ (conjugated to the effective volume $V_{g}$ ) and the chemical potential $\mu$. Heat capacities and compressibilities follow from second derivatives of the thermodynamical potentials (e.g. [46]). The expression of $P_{g}$ is

$$
P_{g} V_{g}\left(1+\frac{\sqrt{\pi}}{2} \frac{A_{h}}{V_{g}} L+\frac{a_{1}}{V_{g}} L^{2}+\frac{b_{1}}{V_{g}} L^{3}+\frac{a_{2}}{V_{g}} L^{4}+\ldots\right)=\frac{\mathrm{N} M_{o} \alpha_{o}^{2}}{2 L^{2}},
$$

or, perhaps more transparently, in terms of $T$

$$
P_{g} V_{g}\left(1+\frac{\sqrt{\pi}}{2} \frac{A_{h}}{V_{g}}\left(\frac{M_{o} \alpha_{o}^{2}}{2 k_{\mathrm{B}} T}\right)^{\frac{1}{2}}+\frac{a_{1}}{V_{g}}\left(\frac{M_{o} \alpha_{o}^{2}}{2 k_{\mathrm{B}} T}\right)+\frac{b_{1}}{V_{g}}\left(\frac{M_{o} \alpha_{o}^{2}}{2 k_{\mathrm{B}} T}\right)^{\frac{3}{2}}+\ldots\right)=\mathrm{N} k_{\mathrm{B}} T .
$$

The corrections in $V_{g}=V_{g}(T)$ to the ideal gas equation of state indicate the presence of effective interactions due to stratification and topography, encoded in curvature and boundary terms in the heat kernel coefficients (a real fluid modelled e.g. with Van der Waals equation of state would also have $P_{g}=P_{g}(T)$ corrections). It also follows for $\mu$

$$
\mu=k_{\mathrm{B}} T \ln \left(\frac{\mathrm{N}}{V_{g}(T)}\left(\frac{2 \pi M_{o} \alpha_{o}^{2}}{k_{\mathrm{B}} T}\right)^{\frac{3}{2}}\right) .
$$

The rest of thermodynamical quantities follow similarly. Particularly important is the relation between the energy $E$ and temperature $T$ (or alternatively, the length scale $L$ )

$$
\begin{aligned}
E & =\langle E\rangle=-\frac{\partial}{\partial \beta} \ln Z \\
& =\frac{3}{2} \mathrm{~N} k_{\mathrm{B}} T\left(1-\frac{\sqrt{\pi}}{6} \frac{A_{h}}{V_{g}(T)}\left(\frac{M_{o} \alpha_{o}^{2}}{2 k_{\mathrm{B}} T}\right)^{\frac{1}{2}}-\frac{2}{3} \frac{a_{1}}{V_{g}(T)}\left(\frac{M_{o} \alpha_{o}^{2}}{2 k_{\mathrm{B}} T}\right)-\frac{b_{1}}{V_{g}(T)}\left(\frac{M_{o} \alpha_{o}^{2}}{2 k_{\mathrm{B}} T}\right)^{\frac{3}{2}}+\ldots\right) .
\end{aligned}
$$

In particular, rewriting it in terms of $L$ and using (35) for $E$ and (E.1) for $\mathrm{N}$

$$
\frac{2}{3} \cdot \frac{\int_{M}|\nabla \psi|^{2} d V_{g}}{\int_{M}|\psi|^{2} d V_{g}}=\frac{1}{L^{2}}\left(1-\frac{\sqrt{\pi}}{6} \frac{A_{h}}{V_{g}(L)} L-\frac{2}{3} \frac{a_{1}}{V_{g}(L)} L^{2}-\frac{b_{1}}{V_{g}(L)} L^{3}+\ldots\right) .
$$

This provides a recursive relation to determine the length scale $L$, that includes stratification and topography terms and recovers $甘 L$ in (68) as the zeroth-order.

Appendix E.2.1. Equilibrium condition: first derivatives of the potentials. Mechanical, chemical and thermal equilibria between subsystems $A$ and $B$, are characterized by the equality of corresponding intensive variables, respectively $P_{g}, \mu$ and $T$ (or $L$ ), namely

$$
\left(P_{g}\right)_{A}=\left(P_{g}\right)_{B} \quad, \quad \mu_{A}=\mu_{B} \quad, \quad T_{A}=T_{B} .
$$

If these conditions are not satisfied, a change/transfer of effective volume $V_{g}$, particles $\mathrm{N}$ or "heat" happen between subsystems $A$ and $B$. In other words, $\int_{A, B} 1 d V_{g}, \int_{A, B}|\psi|^{2} d V_{g}$ and/or $\int_{A, B}|\nabla \psi|_{g}^{2} d V_{g}$ must change so that $P_{g}, \mu$ and $T$ balance. Extrapolating these

$\sharp$ This justifies the factor $\frac{2}{3}$ in the definition (68). Alternatively, it could have been included in (E.5). 
An Ocean Drum: quasi-geostrophic energetics from a Riemann geometry perspective 28 toy-model results as qualitative indications in the $L \ll L_{\max }^{\text {basin }}$ regime of the actual QG model, quantities $P_{g}, \mu$ and $T$ become functionals of $\psi$ providing information about the contact between water masses. This is the approach to diagnostic tools adopted in 5.3 .3 .

Appendix E.2.2. Stability conditions: second derivatives of the potentials. Thermodynamical stability conditions impose sign conditions on certain second derivatives of thermodynamical potentials. For instance, for the free energy $F$ it must hold [46]

$$
\left(\frac{\partial^{2} F}{\partial T^{2}}\right)_{V_{g}, \mathrm{~N}} \leq 0,\left(\frac{\partial^{2} F}{\partial V_{g}^{2}}\right)_{T, \mathrm{~N}} \geq 0 .
$$

The violation of such stability constraints signals the conditions for a phase transition: the system must re-adapt its internal structure into new configurations reducing the free energy, possibly incorporating new elements in its physical description. Extrapolating again such toy-model results to the proper QG model (caveats regarding non-extensitivity in long-range interacting systems are particularly relevant here), this provides another kind of diagnostics with interest in the assessment of stratification and topography in the transition from the $\mathrm{QG}$ regime to other dynamical ocean regimes. The condition $L \ll L_{\max }^{\text {basin }}$ in the toy-model suggests the focus on the transition to submesoscale physics (though a transition to large-scale ocean dynamics could be envisaged for a sufficiently large basin size). For concreteness, let us mention the stability condition provided by the positivity of the heat capacity at constant volume $C_{V}$

$$
C_{V} \equiv\left(\frac{\partial E}{\partial T}\right)_{V_{g}, \mathrm{~N}} \geq 0
$$

providing another diagnostic functional on $\psi$ (its expression can be derived from (E.17)).

Appendix E.2.3. Statistical fluctuations. Statistical mechanics provides directly other flow estimators, e.g. statistical fluctuations (closely related to phase transitions). The grand-canonical partition function $\Xi=\Xi\left[T, V_{g}, \mu\right]$ in (E.11) can be expressed as

$$
P_{g} V_{g}=k_{\mathrm{B}} T \ln \Xi \text {. }
$$

The expression for $P_{g}=P_{g}\left(T, V_{g}, \mu\right)$ can be obtained from (E.15) and (E.16). The fluctuation in the number of particles in the grand-canonical ensemble has the form

$$
(\delta \mathrm{N})^{2}=\left\langle\mathrm{N}^{2}\right\rangle-\langle\mathrm{N}\rangle^{2}=\left(\frac{\partial^{2} \ln \Xi}{\partial(\beta \mu)^{2}}\right)_{\left(T, V_{g}\right)},
$$

from which we obtain

$$
(\delta \mathrm{N})^{2}=\left(\frac{2 \pi M_{o} \alpha_{o}^{2}}{k_{\mathrm{B}} T}\right)^{-\frac{3}{2}} e^{\frac{\mu}{k_{\mathrm{B}} T}} V_{g}=\frac{1}{(4 \pi)^{\frac{3}{2}}}\left(\frac{V_{g}}{L^{3}}\right) e^{\left(\frac{2}{M_{o} \alpha^{2}}\right) \mu L^{2}} .
$$

We note that topography and stratification do not enter in $(\delta \mathrm{N})^{2}$, since the latter is independent of heat kernel coefficients (dependence on $\alpha_{o}$ is inherited from the definition of $\mathrm{N}$ ). Fluctuations in the energy in the canonical ensemble are likewise expressed as

$$
(\delta E)^{2}=\left\langle E^{2}\right\rangle-\langle E\rangle^{2}=\left(\frac{\partial^{2} \ln Z}{\partial \beta^{2}}\right)_{\left(V_{g}, \mathrm{~N}\right)} .
$$


An Ocean Drum: quasi-geostrophic energetics from a Riemann geometry perspective 29

Explicit expressions can be obtained from (E.9). Quantities $(\delta \mathrm{N})^{2}$ and $(\delta E)^{2}$ can be used as functionals of $\psi$ to diagnose zones of intense activity in the QG flow.

Appendix E.2.4. A comment on the relation to full $Q G$ statistical mechanics. The statistical mechanics approach to QG dynamics is the subject of an extensive research effort. Comprehensive accounts of the topic can be found in [19, 47]. In particular, the RSM theory [13, 14, 15, 16] incorporates all dynamical invariants (10) in its treatment and provides a sound theoretical framework where previous approaches (in particular for 2-dimensional Euler flows) are included as appropriate limits [19]. This theory not only explains successfully qualitative properties of geophysical fluids (in particular with applications in the ocean, cf. [19]), but also provides a full quantitative approach to the QG dynamics problem, namely aiming at the description of the most probable final state by identifying the relevant macrostates and their probability (note the difference between this quantitative goal and the Cauchy problem resolution in PDE approaches).

In contrast with the RSM theory, the statistical mechanics toy-model discussed here only considers the conservation of energy, ignoring all other dynamical invariants. The elimination of the corresponding long-range interactions entails key physical differences with the QG model, in particular the extensitivity of the energy in (E.17), not realized in fluid dynamical models as the QG one. It also impacts the possible non-equivalence of statistical ensembles, in particular the physical suitability of the microcanonical ensemble in fluid dynamics over the canonical ensemble (e.g. [17]). Our model, explicitly built on the (fluid non-appropriate) canonical ensemble, ignores this issue. For these reasons $\dagger$, the discussed toy-model is not meant to address the physical dynamics of QG flows, as e.g. RSM theory does. Rather than explaining and/or predict, the model attempts to provide a set of systematic tools to monitor data of QG flows in a first rough analysis, identifying regions where finer and more powerful tools can be applied.

Having said this, the toy-model can provide some interesting insights. On the one hand, the exponential decay of QG flow interactions in scales much larger than the internal Rossby deformation radius [48, 45], makes the toy-model a progressively better physical approximation at large distances (of interest precisely in the $L \ll L_{\max }^{\text {basin }}$ regime). In particular, (finite volume) heat-kernel coefficient corrections might account for actual physical QG finite volume effects. On the other hand, the underlying Fock representation may suggest the avenue to a (physically realistic) statistical field theory, complementary to the RSM mean-field theory (and possibly relevant for the coupling with fast modes in [40, 41]). This can open a path to import spectral geometry expertise in quantum field theory (e.g. [49]). Finally, formal treatments of the QG model often study barotropic QG equations. The present geometric discussion recasts the full continuous QG model in a form particularly close to the 2-dimensional Euler equations, since the (potential) vorticity is also controlled by an exact Laplacian. This can help to mimic the strategy

†† Another issue concerns the choice of $\alpha_{o}$ in (42), due to the phenomenological absence of a minimal QG length (that can be related to $\alpha_{o}$ ). However, the thermodynamical description offers results independent of $\alpha_{o}$, once expressed in terms of $L$. Therefore $\alpha_{o}$ can be seen as an intermediary technical tool. 
An Ocean Drum: quasi-geostrophic energetics from a Riemann geometry perspective 30

for 2-dimensional Euler flows, in particular extending large deviation results in [17].

\section{References}

[1] Gill, A.: Atmosphere-Ocean Dynamics. International Geophysics. Academic Press, Cambridge, MA, USA (1982)

[2] Pedlosky, J.: Geophysical Fluid Dynamics, 2nd edn. Springer-Verlag, New York, NY, USA (1990)

[3] Salmon, R.: Lectures on Geophysical Fluid Dynamics. Oxford University Press, USA (1998)

[4] Vallis, G.K.: Atmospheric and Oceanic Fluid Dynamics. Cambridge University Press, Cambridge, U.K. (2006)

[5] Cushman-Roisin, B., Beckers, J.: Introduction to Geophysical Fluid Dynamics: Physical and Numerical Aspects. Academic Press, Cambridge, MA, USA (2011).

[6] Ferrari, R., Wunsch, C.: Ocean circulation kinetic energy: Reservoirs, sources, and sinks. Annual Review of Fluid Mechanics 41(1), 253-282 (2009)

[7] Charney, J.G.: Geostrophic turbulence. J. Atmos. Sci. 28(1), 1087-1095 (1971)

[8] Rhines, P.: Geostrophic turbulence. Annual Review of Fluid Mechanics 11, 401-441 (1979)

[9] Salmon, R.: Geostrophic turbulence. In: A.R.O..P.M. Rizzoli (ed.) Topics in Ocean Physics, pp. 30-78 (1982). Proceedings of the International School of Physics Enrico Fermi (July 1980), Course LXXX

[10] Kraichnan, R.: Inertial ranges in 2 dimensional turbulence. Phys. Fluids 10, 1417 (1967)

[11] Leith, C.: Diffusion approximation for two-dimensional turbulence. Phys. Fluids 11, 671-673 (1968)

[12] Batchelor, G.: Computation of the energy spectrum in homogeneous two-dimensional turbulence. Phys. Fluids 12(II), 233-239 (1969)

[13] Robert, R.: Etats d'équilibre statistique pour l'écoulement bidimensionnel d'un fluide parfait. C. R. Acad. Sci. Paris Ser. I 311, 575-578 (1990)

[14] Miller, J.: Statistical mechanics of Euler equations in two dimensions. Phys. Rev. Lett. 65, 2137$2140(1990)$

[15] Robert, R.: A maximum-entropy principle for two-dimensional perfect fluid dynamics. Journal of Statistical Physics 65(3-4), 531-553 (1991)

[16] Robert, R., Sommeria, J.: Statistical equilibrium states for two-dimensional flows. J. Fluid Mech. 229, 291-310 (1991)

[17] Bouchet, F., Corvellec, M.: Invariant measures of the 2d Euler and Vlasov equations. Journal of Statistical Mechanics: Theory and Experiment 2010(08), P08,021 (2010)

[18] Majda, A.: Introduction to PDEs and waves for the atmosphere and ocean. Courant Lecture Notes in Math. 9, Amer. Math. Soc, Providence, USA (2003)

[19] Bouchet, F., Venaille, A.: Statistical mechanics of two-dimensional and geophysical flows. Physics Reports 515(5), 227 - 295 (2012)

[20] Chavel, I.: Eigenvalues in Riemannian geometry. Pure and Applied Mathematics. Elsevier, Burlington, MA (1984)

[21] Kac, M.: Can one hear the shape of a drum? The American Mathematical Monthly 73(4), pp. $1-23(1966)$

[22] Giraud, O., Thas, K.: Hearing shapes of drums: Mathematical and physical aspects of isospectrality. Rev. Mod. Phys. 82, 2213-2255 (2010)

[23] Berger, M.: A panoramic view of Riemannian geometry. Springer, Berlin (2003)

[24] Branson, T., Gilkey, P.: The asymptotics of the Laplacian on a manifold with boundary. Comm. Partial Differential Equations 15, 245-272 (1990)

[25] Gilkey, P.: Asymptotic Formulae in Spectral Geometry. Studies in advanced mathematics. CRC Press, Boca Raton, FL, USA (2003)

[26] Berry, M.V., Dennis, M.R.: Boundary-condition-varying circle billiards and gratings: the Dirichlet singularity. Journal of Physics A: Mathematical and Theoretical 41(13), 135,203 (2008) 
An Ocean Drum: quasi-geostrophic energetics from a Riemann geometry perspective 31

[27] Tung, K.K., Welch, W.T.: Remarks on Charney's note on geostropic turbulence. J. Atmos. Sci. 58(14), 2009-2012 (2001)

[28] Paoletti, M.S., Swinney, H.L.: Propagating and evanescent internal waves in a deep ocean model. Journal of Fluid Mechanics 706, 571-583 (2012)

[29] LaCasce, J.: Surface quasigeostrophic solutions and baroclinic modes with exponential stratification. J. Phys. Oceanogr. 42(4), 569580 (2012)

[30] Benguria, R.D.: Spectral problems in spaces of constant curvature, chap. 12, pp. 131-137. World Scientific (2011)

[31] Simon, B.: Semiclassical analysis of low lying eigenvalues. i. non-degenerate minima : asymptotic expansions. Annales de l'Institut Henri Poincaré (A) Physique théorique 38(3), 295-308 (1983)

[32] Ashbaugh, M.S., Benguria, R.D.: Universal bounds for the low eigenvalues of Neumann Laplacians in n dimensions. SIAM J. Math. Anal. 24(3), 557-570 (1993)

[33] Bouchet, F., Simonnet, E.: Random changes of flow topology in two-dimensional and geophysical turbulence. Phys. Rev. Lett. 102, 094,504 (2009)

[34] Venaille, A., Bouchet, F.: Statistical ensemble inequivalence and bicritical points for twodimensional flows and geophysical flows. Phys. Rev. Lett. 102, 104,501 (2009)

[35] Loxley, P.N., Nadiga, B.T.: Bistability and hysteresis of maximum-entropy states in decaying two-dimensional turbulence. Physics of Fluids 25(1), 015113 (2013)

[36] Clark, C.: The asymptotic distribution of eigenvalues and eigenfunctions for elliptic boundary value problems. SIAM Rev. 9(4), 627-646 (1967)

[37] Balian, R., Bloch, C.: Distribution of eigenfrequencies for the wave equation in a finite domain: I. three-dimensional problem with smooth boundary surface. Annals of Physics 60(2), $401-447$ (1970)

[38] McWilliams, J.C., Yavneh, I., Cullen, M.J.P., Gent, P.R.: The breakdown of large-scale flows in rotating, stratified fluids. Physics of Fluids 10(12), 3178-3184 (1998)

[39] Nadiga, B.T.: Nonlinear evolution of a baroclinic wave and imbalanced dissipation. Journal of Fluid Mechanics 756, 965-1006 (2014)

[40] Young, W.R., Ben Jelloul, M.: Propagation of near-inertial oscillations through a geostrophic flow. J. Mar. Res. 55(4), 735766 (1997)

[41] Xie, J.H., Vanneste, J.: A Generalised-Lagrangian-Mean model of the interactions between nearinertial waves and mean flow. Journal of Fluid Mechanics 774, 143-169 (2015)

[42] Wald, R.M.: General Relativity. Chicago University Press (1984)

[43] Holm, D.D., Zeitlin, V.: Hamilton's principle for quasigeostrophic motion. Physics of Fluids 10(4), 800-806 (1998)

[44] Campa, A., Dauxois, T., Ruffo, S.: Statistical mechanics and dynamics of solvable models with long-range interactions. Physics Reports 480(3-6), 57 - 159 (2009)

[45] Campa, A., Dauxois, T., Dauxois, T., Fanelli, D., Ruffo, S.: Physics of Long-Range Interacting Systems. Oxford University Press (2014)

[46] Callen, H.B.: Thermodynamics and an Introduction to Thermostatistics. Wiley, New York (1985)

[47] Majda, A., Wang, X.: Nonlinear Dynamics and Statistical Theories for Basic Geophysical Flows. Cambridge University Press (2006)

[48] Venaille, A., Bouchet, F.: Solvable phase diagrams and ensemble inequivalence for two-dimensional and geophysical turbulent flows. Journal of Statistical Physics 143(2), 346-380 (2011)

[49] Fursaev, D., Vassilevich, D.: Operators, Geometry and Quanta: Methods of Spectral Geometry in Quantum Field Theory. Theoretical and Mathematical Physics. Springer (2011) 